\title{
W43: the closest molecular complex of the Galactic bar?`
}

\author{
Q. Nguyễn Lương ${ }^{1}$, F. Motte ${ }^{1}$, F. Schuller ${ }^{2}$, N. Schneider ${ }^{1}$, S. Bontemps ${ }^{3}$, P. Schilke ${ }^{4}$, K. M. Menten ${ }^{2}$,

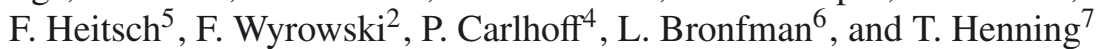

\author{
${ }^{1}$ Laboratoire AIM Paris-Saclay, CEA/IRFU, CNRS/INSU, Université Paris Diderot, Service d'Astrophysique, Bât. 709, \\ CEA-Saclay, 91191 Gif-sur-Yvette Cedex, France \\ e-mail: quang.nguyen-luong@cea.fr \\ 2 Max-Planck-Institut für Radioastronomie, Auf dem Hügel 69, 53121 Bonn, Germany \\ 3 OASU/LAB-UMR 5804, CNRS/INSU, Université Bordeaux 1, 2 rue de l'Observatoire, BP 89, 33270 Floirac, France \\ ${ }^{4}$ I. Physikalisches Institut, Universität zu Köln, Zülpicher Str. 77, 50937 Köln, Germany \\ 5 Department of Physics and Astronomy, University of North Carolina Chapel Hill, Phillips Hall, Chapel Hill, NC 27599-3255, USA \\ 6 Departamento de Astronomia, Universidad de Chile, Casilla 36-D, Santiago, Chile \\ 7 Max Planck Institute for Astronomy, Koenigstuhl 17, 69117 Heidelberg, Germany
}

Received 6 December 2010 / Accepted 14 February 2011

\section{ABSTRACT}

\begin{abstract}
Context. In the framework of the multi-wavelength Galactic surveys of star-formation presently underway, complexes of molecular clouds that stretch over up to hundreds of parsecs are of particular interest. This is because a large population of stars is forming within them, thus all at the same distance from the Sun and under similar physical conditions.

Aims. We study the Galactic plane between $\approx 29.5$ and 31.5 degrees of longitude, which is especially rich in terms of molecular clouds and star-formation activity. It is located within what is sometimes called the molecular ring and contains the Galactic mini-starburst region W43, as well as the prominent hot core G29.96-0.02 with its associated compact HII region.

Methods. We used a large database extracted from Galaxy-wide surveys of $\mathrm{HI},{ }^{13} \mathrm{CO} 1-0,8 \mu \mathrm{m}$, and $870 \mu \mathrm{m}$ continuum to trace diffuse atomic gas, low- to medium-density molecular gas, high-density molecular gas, and star-formation activity, which we complemented by dedicated ${ }^{12} \mathrm{CO} 2-1,3-2$ observations of the region.

Results. From the detailed 3D (space-space-velocity) analysis of the molecular and atomic cloud tracers through the region and despite its wide velocity range $\left(F W H M \sim 22.3 \mathrm{~km} \mathrm{~s}^{-1}\right.$ around $V_{\mathrm{LSR}} \sim 95.9 \mathrm{~km} \mathrm{~s}^{-1}$ ), we identified W43 as a large (equivalent diameter $\sim 140 \mathrm{pc}$ ) and coherent complex of molecular clouds that is surrounded by an atomic gas envelope (equivalent diameter $\sim 290 \mathrm{pc}$ ). We measured both the total mass of this newly identified molecular complex $\left(M_{\text {total }} \sim 7.1 \times 10^{6} M_{\odot}\right)$ and the mass contained in dense $870 \mu \mathrm{m}$ clumps $\left(<5 \mathrm{pc}\right.$ dense cloud structures, $\left.M_{\text {clumps }} \sim 8.4 \times 10^{5} M_{\odot}\right)$, and conclude that W43 is particularly massive and concentrated. The distance that we assume for the W43 complex is $6 \mathrm{kpc}$ from the Sun, which may place it at the meeting point of the Scutum-Centaurus (or Scutum-Crux) Galactic arm and the bar, a dynamically complex region where high-velocity streams could easily collide. We propose that the star-formation rate of W43 is not steady but increases from $\sim 0.01 M_{\odot} \mathrm{yr}^{-1}$ (measured from its $8 \mu \mathrm{m}$ luminosity) to $\sim 0.1 M_{\odot} \mathrm{yr}^{-1}$ (measured from its molecular content). From the global properties of W43, we claim that it is an extreme molecular complex in the Milky Way and it might even form starburst clusters in the near future.

Conclusions. W43 is the perfect testbed to investigate (1) the star-formation process occuring through bursts as well as (2) the formation of such an extreme complex in the framework of converging flows scenarios.
\end{abstract}

Key words. stars: formation - ISM: clouds - submillimeter: ISM - HII regions - dust, extinction - ISM: kinematics and dynamics

\section{Introduction}

Molecular clouds forming low-mass stars in our solar neigborhood have been well known and are mostly located in the Gould Belt, a giant coherent cloud structure inclined to the Galactic plane (e.g. Comerón \& Torra 1994). High-mass stars should form in complexes of giant molecular clouds (GMCs) also called giant molecular associations (GMAs), i.e., groups of molecular clouds with a total mass maybe up to $10^{7} M_{\odot}$ (Rand \& Kulkarni 1990; Rand 1993; Kuno et al. 1995; Koda et al. 2009). While a few molecular complexes have been recognized to be forming high-mass stars in the outer Galaxy (e.g. Cygnus $\mathrm{X}$ and, in a more modest form, NGC 7538), finding coherent associations of high-mass star-forming regions on a Galactic scale has been challenging. The main reason for this is that near the Galactic

\footnotetext{
* Appendix A is only available in electronic form at http://www. aanda.org
}

equator, where these massive complexes are expected to reside, the identification of molecular complexes and GMAs is confused with other line-of-sight clouds. Galaxy-wide low-resolution $\mathrm{CO}$ surveys have provided lists of molecular cloud groups that are often interpreted as consisting of a blend of clouds spread along the line of sight (e.g. Dame et al. 2001). We hereafter refer to W43 as the emission threading the region $l=\left(29^{\circ}-32^{\circ}\right)$, $b=\left(-1^{\circ}-+1^{\circ}\right)$ of the Galactic plane. W43 has been found to contain two of the largest cloud groups of the first Galactic quadrant with a total mass of $\sim 5 \times 10^{6} M_{\odot}$ (close to $l=31^{\circ}$ and line-of-sight velocity, $V_{\mathrm{LSR}}=95 \mathrm{~km} \mathrm{~s}^{-1}$, and close to $l=29^{\circ}$ and $V_{\mathrm{LSR}}=80 \mathrm{~km} \mathrm{~s}^{-1}$; Dame et al. 1986). Solomon et al. (1987) used higher resolution $\mathrm{CO}$ surveys to distinguish the ensemble of W43 clouds into 14 clouds and locate the 2 most massive ones: SRBY 162 (which we call W43-main) and SRBY 171 (which is called W43-south), which have virial masses of several times $10^{6} M_{\odot}$ (see also Liszt 1995; Mooney et al. 1995). This is 
Table 1. Observational parameters of the tracers used in the present paper.

\begin{tabular}{lllllll}
\hline \hline $\begin{array}{l}\text { Telescope } \\
\text { SSurvey }\end{array}$ & Tracer & $\begin{array}{l}\text { Frequency } \\
{[\mathrm{GHz}]}\end{array}$ & $\begin{array}{l}\text { HPBW } \\
{[\operatorname{arcsec}]}\end{array}$ & $\begin{array}{l}\text { Velocity range } \\
{\left[\mathrm{km} \mathrm{s}^{-1}\right]}\end{array}$ & $\begin{array}{l}\Delta v_{\text {res }} \\
{\left[\mathrm{km} \mathrm{s}^{-1}\right]}\end{array}$ & $1 \sigma$ rms \\
\hline VLA/VGPS & H I & 1.420 & $60^{\prime \prime}$ & -120 to 170 & 1.56 & $1.80 \mathrm{~K} \mathrm{~km} \mathrm{~s}^{-1}$ \\
CfA/CfA & ${ }^{12} \mathrm{CO} \mathrm{1-0}$ & 115.271 & $450^{\prime \prime}$ & -0.5 to 271 & 0.65 & $0.22 \mathrm{~K} \mathrm{~km} \mathrm{~s}^{-1}$ \\
KOSMA/present paper & ${ }^{12} \mathrm{CO} 2-1$ & 230.537 & $130^{\prime \prime}$ & 0 to 200 & 0.20 & $0.11 \mathrm{~K} \mathrm{~km} \mathrm{~s}^{-1}$ \\
KOSMA/present paper & ${ }^{12} \mathrm{CO} \mathrm{3-2}$ & 345.796 & $80^{\prime \prime}$ & 0 to 200 & 0.30 & $0.52 \mathrm{~K} \mathrm{~km} \mathrm{~s}^{-1}$ \\
FCRAO/GRS & ${ }^{13} \mathrm{CO} 1-0$ & 110.201 & $46^{\prime \prime}$ & -5 to 135 & 0.21 & $0.13 \mathrm{~K} \mathrm{~km} \mathrm{~s}^{-1}$ \\
APEX/ATLASGAL & $870 \mu$ m continuum & $\ldots$ & $19^{\prime \prime}$ & $\ldots$ & $\ldots$ & $60 \mathrm{mJy} \mathrm{beam}^{-1}$ \\
SPITZER/GLIMPSE & $8 \mu \mathrm{m}$ continuum & $\ldots$ & $6^{\prime \prime}$ & $\ldots$ & $\ldots$ & $0.08 \mathrm{mJy} \mathrm{beam}^{-1}$ \\
\hline
\end{tabular}

confirmed by Rathborne et al. (2009) who used ${ }^{13} \mathrm{CO}$ emission as a higher density tracer observed in the higher resolution Galactic ring survey (GRS) to identify more than 20 molecular clouds in the $\mathrm{W} 43$ region, with systemic velocities ranging from $12 \mathrm{~km} \mathrm{~s}^{-1}$ to $110 \mathrm{~km} \mathrm{~s}^{-1}$. The core of W43-main harbors a well-known giant HII region powered by a particularly luminous cluster of Wolf-Rayet (WR) and OB stars $\left(\sim 3.5 \times 10^{6} L_{\odot}\right.$, see Blum et al. 1999, and references therein). Motte et al. (2003) established it as a dense region equivalent to a mini-starburst region since it is undergoing a remarkably efficient episode of high-mass starformation (the discovery of $\sim 15$ high-mass protoclusters leading to star-formation efficiency ( $S F E$ ) of $\sim 25 \% / 10^{6} \mathrm{yr}$ ). Far-infrared to submillimeter data from the Herschel Space Observatory revealed a complex structure of chimneys and filaments, and confirmed its efficiency in forming massive stars (Bally et al. 2010). W43-south corresponds to a less extreme cloud, which however, harbors the well-known compact HII (CHII) region ${ }^{1}$. The environment of W43-main and W43-south needs to be investigated in greater detail to determine whether these GMCs could be related and form an individual complex or a GMA.

The APEX ${ }^{2}$ Telescope Large Area Survey of the GALaxy (ATLASGAL, Schuller et al. 2009) has made an unbiased census of young stellar objects deeply embedded in the GMCs. The survey of the inner Galactic plane including the W43 region displays a rich collection of compact sources within W43, which correspond to the high-density backbones of molecular clouds.

Before making a meaningful assessment of the global characteristics of the star-forming regions, a strong effort is needed to identify GMCs and groups of GMCs which we could call a molecular complex or GMA. In this paper, we define the W43 molecular complex and then determine its global characteristics. Employing the large database described in Sect. 2, we show that W43 is a coherent molecular and star-forming complex surrounded by atomic gas (see Sect. 3). In Sect. 4, we locate W43 at the connecting point between the Scutum-Centaurus arm and the Galactic bar and quantify its mass and star-formation activity. Section 5 concludes that W43 is indeed an extreme molecular cloud and star-forming complex of our Galaxy, located only $\sim 6 \mathrm{kpc}$ from the Sun.

\footnotetext{
${ }^{1}$ G29.96-0.02 is normally termed an ultracompact HII region. However, we note that its size and radio flux density resembles that of the Orion Nebula, the archetypical CHII region, when the latter is placed at a distance of $6 \mathrm{kpc}$. G29.96-0.02 is excited by at least one O star (Cesaroni et al. 1998; Pratap et al. 1999; Beuther et al. 2007).

2 APEX, the Atacama Pathfinder EXperiment, is a collaboration between the Max Planck Institut für Radioastronomie, the Onsala Space Observatory, and the European Southern Observatory. Images are available at http://www.mpifr-bonn.mpg.de/div/atlasgal/
}

\section{Observations and database}

We used data from different Galactic plane surveys and performed additional observations of the W43 region. These data sets are summarized in Table 1.

\subsection{Hı database}

The atomic gas data of W43 are taken from the Very Large Array (VLA) Galactic Plane Survey (VGPS ${ }^{3}$, Stil et al. 2006). This is a survey of the $21 \mathrm{~cm}$ continuum and line emission from neutral atomic hydrogen, HI, performed through the Galactic plane $\left(18^{\circ}<l<67^{\circ},|b|<1.3^{\circ}\right)$ by the VLA and combined with shortspacing information obtained with the Green Bank Telescope (GBT). This data set has a spatial resolution of $1^{\prime} \times 1^{\prime}$ and a velocity resolution of $1.56 \mathrm{~km} \mathrm{~s}^{-1}$, spanning the range -120 to $170 \mathrm{~km} \mathrm{~s}^{-1}$.

\section{2. ${ }^{12} \mathrm{CO}$ observations and ${ }^{13} \mathrm{CO}$ database}

To trace the low-density molecular gas, we used the ${ }^{12} \mathrm{CO} 1-0$ and ${ }^{13} \mathrm{CO} 1-0$ data from previous Galactic plane surveys and, in addition, observed the ${ }^{12} \mathrm{CO} 2-1$ and ${ }^{12} \mathrm{CO} 3-2$ lines with the 3 m diameter Kölner Observatorium für SubMm-Astronomie $\left(\mathrm{KOSMA}^{4}\right.$ ) from 2009 January to February.

The ${ }^{12} \mathrm{CO} 1-0$ data set is taken from the Galactic plane survey made with the CfA $1.2 \mathrm{~m}^{\text {telescope }}{ }^{5}$ (Dame et al. 2001). These data have a spatial resolution of $450^{\prime \prime}$ and a velocity resolution of $0.65 \mathrm{~km} \mathrm{~s}^{-1}$ spanning the velocity range -0.5 to $271 \mathrm{~km} \mathrm{~s}^{-1}$.

We also used the ${ }^{13} \mathrm{CO} 1-0$ data of the Boston UniversityFive College Radio Astronomy Observatory (BU-FCRAO) Galactic ring survey (GRS) ${ }^{6}$. This survey was performed with the FCRAO $14 \mathrm{~m}$ telescope and covers the Galactic plane in the range $18^{\circ}<l<55.7^{\circ}$ for $|b|<1^{\circ}$ (Jackson et al. 2006). It has a spatial resolution of $46^{\prime \prime}$ and a velocity resolution of $0.21 \mathrm{~km} \mathrm{~s}^{-1}$ spanning the velocity range -5 to $135 \mathrm{~km} \mathrm{~s}^{-1}$. The spectral cubes are converted into a main-beam brightness temperature using an

\footnotetext{
3 The full spectral cubes of the VGPS database are available as FITS files at http://www.ras.ucalgary.ca/VGPS/. The VLA and GBT are facilities of the National Radio Astronomy Observatory.

4 The KOSMA 3 m submillimeter telescope was originally installed on Gornergrat-Süd, Switzerland, operated by the University of Cologne in collaboration with the University of Bonn. It has now been rebuilt close to Lhasa/Tibet.

5 The CfA telescope is the $1.2 \mathrm{~m}$ Millimeter-Wave Telescope of the Center for Astrophysics, Harvard University.

${ }^{6}$ The full spectral FITS cubes are available to download at http://www.bu.edu/galacticring
} 


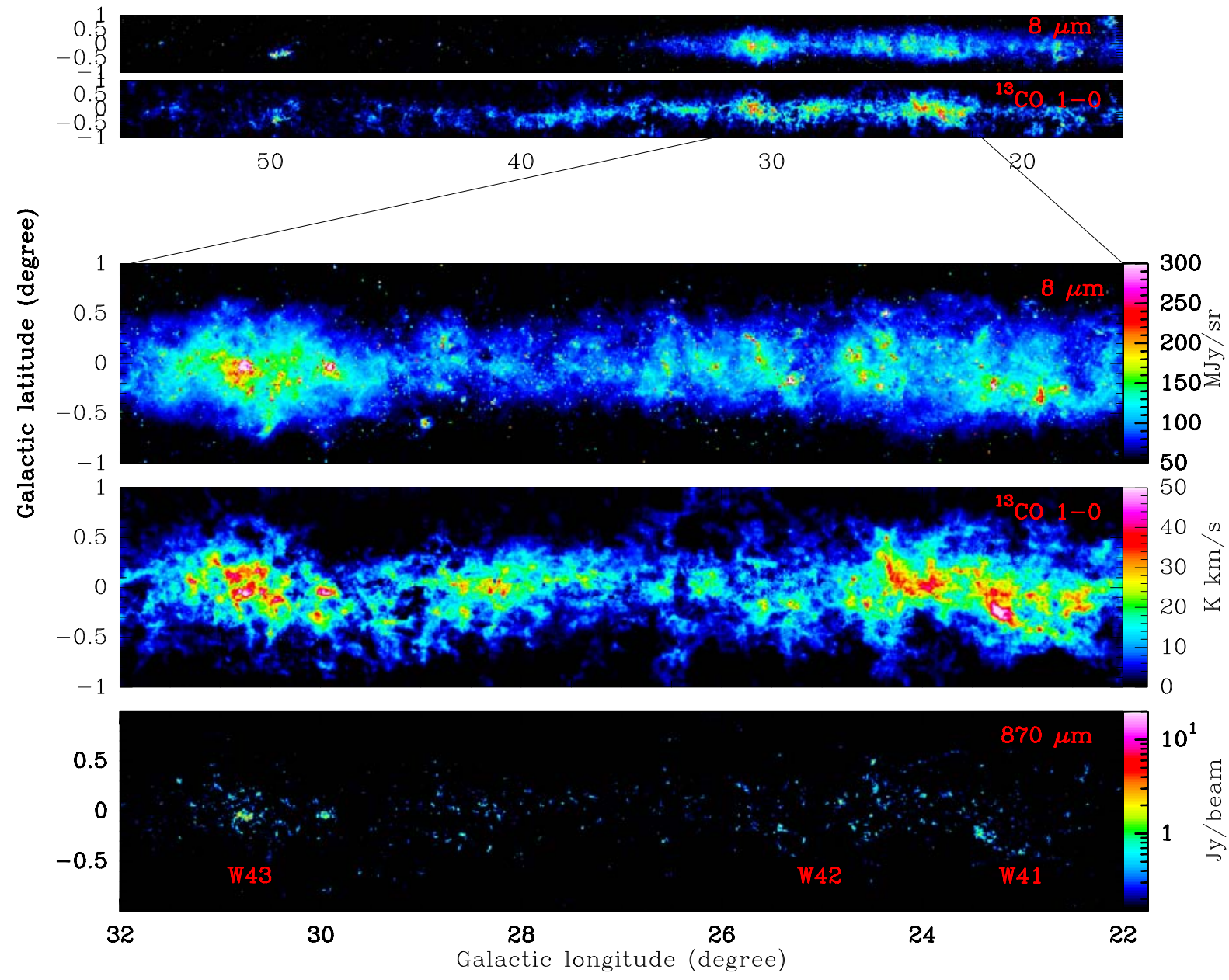

Fig. 1. Galactic plane from $l=18^{\circ}$ to $l=56^{\circ}$ shown: a) at $8 \mu \mathrm{m}$ (Benjamin et al. 2003); b) in the ${ }^{13} \mathrm{CO} 1-0$ line, integrated from -5 to $135 \mathrm{~km} \mathrm{~s} \mathrm{~s}^{-1}$ (Jackson et al. 2006). Zoom on $l=22^{\circ}-32^{\circ}$ : c) at $8 \mu \mathrm{m}$; d) in ${ }^{13} \mathrm{CO} 1-0$; and e) at $870 \mu \mathrm{m}$ (Schuller et al. 2009).

efficiency of 0.48 . This calibration can overestimate the brightness by up to $30 \%$ because it is performed before any correction for stray radiation (Chris Brunt priv. com.).

In addition, observations of ${ }^{12} \mathrm{CO} 2-1$ and ${ }^{12} \mathrm{CO} 3-2$ lines were performed with a dual-channel SIS-receiver, built at KOSMA, operating at 210-270 and 325-365 GHz, which is mounted on the KOSMA telescope (Graf et al. 1998). The spectra were calibrated to a main-beam brightness temperature scale $\left(T_{\mathrm{MB}}\right)$ using the main beam efficiencies $0.72(230 \mathrm{GHz})$ and $0.68(345 \mathrm{GHz})$. Pointing was monitored simultaneously for both receiver channels using continuum cross-scans of Jupiter and found to be accurate to within $15^{\prime \prime}-30^{\prime \prime}$. The calibration accuracy is approximately $20 \%$. We used the on-the-fly observing mode at KOSMA (Kramer et al. 1998) to map and resample areas of $\sim 1^{\circ 2}$ (for ${ }^{12} \mathrm{CO} 2-1$ ) and $\sim 0.5^{\circ 2}$ (for ${ }^{12} \mathrm{CO} 3-2$ ) on $60^{\prime \prime} \times 60^{\prime \prime}$ grids. The angular resolutions at $230 \mathrm{GHz}$ and at $345 \mathrm{GHz}$ are $130^{\prime \prime}$ and $80^{\prime \prime}$, respectively. Figures A1-A4 show the integrated and channel maps of ${ }^{12} \mathrm{CO} 2-1$ and ${ }^{12} \mathrm{CO} 3-$ 2 emissions obtained with KOSMA. We note that only the region around W43-main was mapped in the ${ }^{12} \mathrm{CO} 3-2$ emission line.

\subsection{Dust continuum image at $870 \mu \mathrm{m}$}

ATLASGAL provides an image of the $870 \mu \mathrm{m}$ dust continuum emission of W43 as part of its coverage of the inner Galactic plane $\left(-60^{\circ}<l<60^{\circ}\right.$ for $\left.|b|<1-1.5^{\circ}\right)$ imaging. This survey was completed with the Large APEX Bolometer Camera Array (LABOCA) installed at the $12 \mathrm{~m}$ APEX telescope located on Llano Chajnantor in Chile. Its bandpass is centered at $345 \mathrm{GHz}$ and it has a bandwidth of $60 \mathrm{GHz}$. The FWHM at this frequency is $\sim 19^{\prime \prime}$ and the mean rms noise level in the W43 region is $\sim 60 \mathrm{mJy}^{-1} \mathrm{bem}^{-1}$. The data reduction is described in Schuller et al. (2009) and is continuously being improved. In the ATLASGAL images, most of the emission that appears uniform on scales larger than $6^{\prime}$ is effectively filtered out. At $6 \mathrm{kpc}$ and above the $5 \sigma$ level, the ATLASGAL survey traces $>80 M_{\odot}$ compact cloud fragments (see Eq. (4)) that have sizes of $<5 \mathrm{pc}$, thus volume densities of $>2 \times 10^{3} \mathrm{~cm}^{-3}$.

\subsection{Mid-infrared database}

W43 was imaged by the Spitzer satellite as part of the Legacy Science Program GLIMPSE (Galactic Legacy Infrared MidPlane Survey Extraordinaire infrared survey), which covers the Galactic plane $\left(-65^{\circ}<l<65^{\circ}\right.$ for $|b|<1^{\circ}$, Benjamin et al. 2003). The emission in the Spitzer/IRAC band 4 at $8 \mu \mathrm{m}$ is dominated by emission of polycyclic aromatic hydrocarbon (PAH) heated by UV photons and is thus related to the "present" starformation activity (e.g. Peeters et al. 2004). 


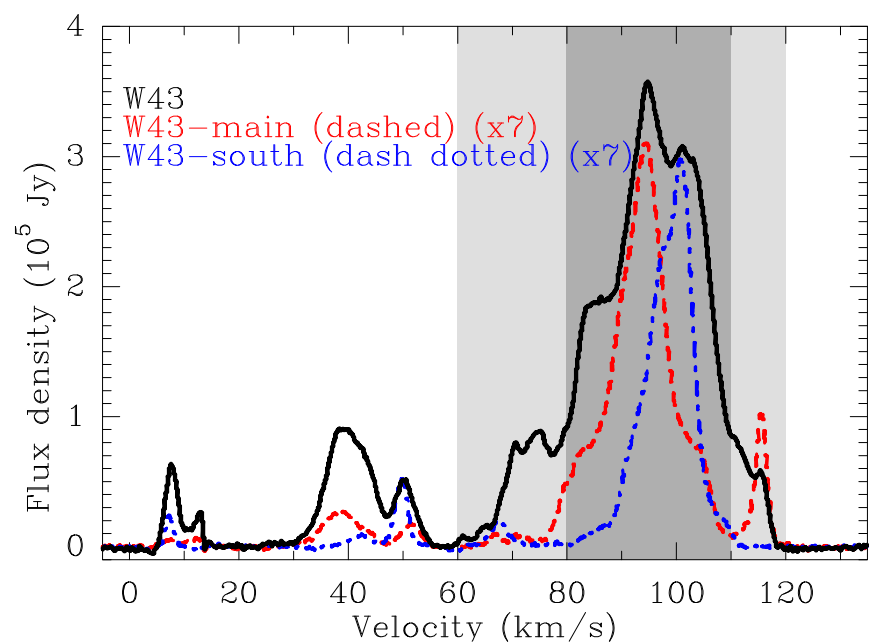

Fig. 2. ${ }^{13} \mathrm{CO} 1-0$ line spectra resulting from the sum of all spectra observed in the $1.8^{\circ} \times 0.8^{\circ}$ area covering W43 (black line), compared to those summed in the circular areas shown in Fig. 4 to be associated with W43-main (red dashed line) and W43-south (blue dashed-dotted line). The main and complete velocity ranges of W43 (see text) are indicated with light- and dark-gray filling.

\section{Results and analysis}

\subsection{W43: a prominent region of the first Galactic quadrant}

High angular resolution surveys of the Galactic plane have generally focused on the $-60^{\circ}<l<60^{\circ}$ longitude range (e.g. ATLASGAL and GLIMPSE) or even avoided the Galactic center as in the GRS survey $\left(18^{\circ}<l<56^{\circ}\right)$. Figure 1 presents the Galactic plane from $l=18^{\circ}$ to $56^{\circ}$ as seen in different surveys, tracing the column density of molecular clouds $\left({ }^{13} \mathrm{CO}\right)$, their star-formation activity $(8 \mu \mathrm{m})$, and the regions of highdensity gas $(870 \mu \mathrm{m})$. In the intensity map in which the ${ }^{13} \mathrm{CO} 1-$ 0 emission is integrated over the complete velocity range it covers (from -5 to $135 \mathrm{~km} \mathrm{~s}^{-1}$, see Fig. 1), W43 indeed stands out as one of the largest and brightest groups of extended molecular complexes extending over $\sim 2$ degrees around Galactic longitude $l=30.5^{\circ}$. Moreover, in the $870 \mu \mathrm{m}$ and $8 \mu \mathrm{m}$ surveys, which trace dense protostellar material and activity of newly born stars, respectively, W43 is also one of the brightest regions in the first Galactic quadrant (see Fig. 1). With its molecular gas brightness, the concentration of cloud material in dense regions, and its high star-formation activity, W43 is one of the most outstanding complexes in the first quadrant.

\subsection{The main velocity range of $W 43$}

Though the image of the ${ }^{13} \mathrm{CO}$ integrated intensity shows W43 as a prominent region in this portion of the Galactic plane, the velocity range from -5 to $135 \mathrm{~km} \mathrm{~s}^{-1}$ certainly covers clouds lying at various distances along the same line of sight. This becomes obvious when inspecting the ${ }^{13} \mathrm{CO}$ spectrum over the entire W43 complex (the extent defined in Sect. 3.3, see Fig. 3d). Figure 2 presents the ${ }^{13} \mathrm{CO} 1-0$ spectra integrated over the entire W43 region, over W43-main and over W43-south. These accumulated spectra allow us to quantify the total flux density at a specific velocity. In Fig. 2, there are at least three groups of molecular clouds that contribute to the W43 spectrum: in the LSR velocity range $5-15 \mathrm{~km} \mathrm{~s}^{-1}, 30-55 \mathrm{~km} \mathrm{~s}^{-1}$, and $60-120 \mathrm{~km} \mathrm{~s}^{-1}$. In the wider velocity range, the spectrum is very bright from 80 to $110 \mathrm{~km} \mathrm{~s}^{-1}$ and has extensions at $60-80 \mathrm{~km} \mathrm{~s}^{-1}$ and $110-120 \mathrm{~km} \mathrm{~s}^{-1}$. The spatially integrated emission in the

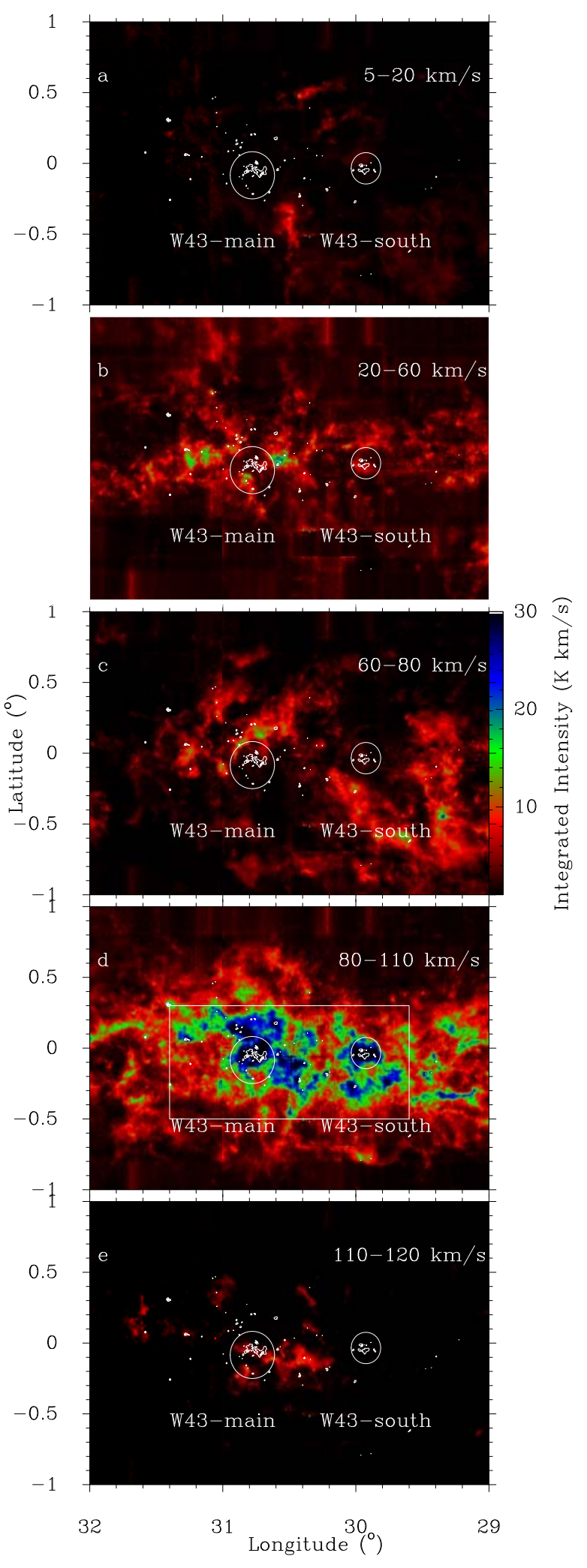

Fig. 3. Integrated maps of the ${ }^{13} \mathrm{CO} 1-0$ line (in color scale) in the direction of the W43 molecular cloud complex whose densest parts are outlined by the $870 \mu \mathrm{m}$ continuum emissions (in contours). The various velocity intervals used for the integration correspond to : a)-b) clouds not associated with W43; c), e) clouds emitting in the low- and highvelocity wings of the W43 line shown in Fig. 2; d) the main velocity range of the W43 molecular complex. The white circles indicate W43main and W43-south, their areas are used to integrate the spectra of Fig. 2. 


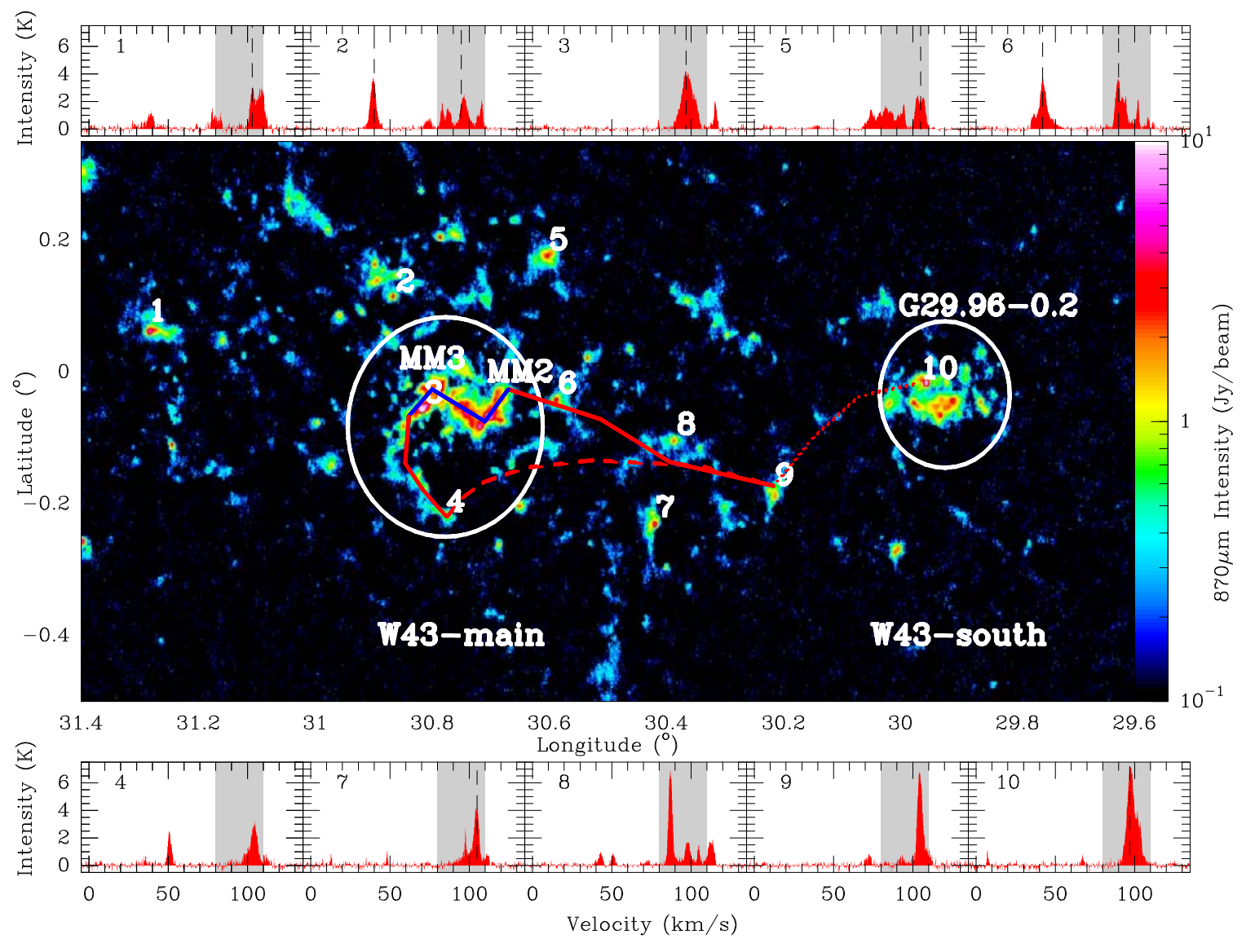

Fig. 4. $870 \mu \mathrm{m}$ continuum emission (color scale) and ${ }^{13} \mathrm{CO} 1-0$ line spectra observed toward a few bright $870 \mu \mathrm{m}$ continuum peaks. In the panels showing spectra taken toward the numbered positions in the image, the main velocity range of $\mathrm{W} 43\left(80-110 \mathrm{~km} \mathrm{~s}^{-1}\right)$ is indicated with gray filling, while dashed lines mark the $\mathrm{NH}_{3}$ peak velocities (Wienen et al., in prep.). In the image, the two main bridges identified between W43-main and South (blue bars) are outlined by red curves, which are continuous when they correspond to ${ }^{13} \mathrm{CO} 1-0$ filaments clearly identified by Motte et al. (in prep.) and dashed or dotted for more tentative connections. W43-MM1, W43-MM2, and G29.96-0.2 are indicated as reference points. The white circles indicate W43-main and W43-south, their areas are used to integrate the spectra of Fig. 2.

velocity ranges $5-15 \mathrm{~km} \mathrm{~s}^{-1}, 30-55 \mathrm{~km} \mathrm{~s}^{-1}, 60-80 \mathrm{~km} \mathrm{~s}^{-1}$, and $110-120 \mathrm{~km} \mathrm{~s}^{-1}$ corresponds to similar total flux densities $\left(\sim 1 \times 10^{5} \mathrm{Jy}\right)$ and is four times weaker than the main line peak at $80-110 \mathrm{~km} \mathrm{~s}^{-1}\left(\sim 3.6 \times 10^{5} \mathrm{Jy}\right)$. We associate the emission in the range $80-110 \mathrm{~km} \mathrm{~s}^{-1}$ with the bulk of the W43 molecular complex and emission from other ranges to either (1) diffuse clouds completely unrelated to W43 $\left(5-15 \mathrm{~km} \mathrm{~s}^{-1}\right.$ and $30-55 \mathrm{~km} \mathrm{~s}^{-1}$ range) or (2) clouds that possibly reside in the envelope of the W43 complex $\left(60-80 \mathrm{~km} \mathrm{~s}^{-1}\right.$ and $\left.110-120 \mathrm{~km} \mathrm{~s}^{-1}\right)$. The integrated maps for these five velocity ranges are given in Figs. 3a-e.

The main velocity range of W43 is defined to be from 80 to $110 \mathrm{~km} \mathrm{~s}^{-1}$, i.e. with an accumulated flux density of W43 larger than $1 / 4 \times S^{\text {peak }} \simeq 1 \times 10^{5}$ Jy. This range is tightly associated with the two densest regions, W43-main and W43-south. The spectrum integrated over the entire W43 is fitted, across the main velocity range of W43 $\left(80-110 \mathrm{~km} \mathrm{~s}^{-1}\right)$, by a Gaussian that peaks at $\sim 95.9 \mathrm{~km} \mathrm{~s}^{-1}$ and has a $F W H M$ of $\sim 22.3 \mathrm{~km} \mathrm{~s}^{-1}$. We first ignored the parts of the ${ }^{13} \mathrm{CO}$ line that are lower than $80 \mathrm{~km} \mathrm{~s}^{-1}$ and higher than $110 \mathrm{~km} \mathrm{~s}^{-1}$ since they are hardly associated with the densest parts of the molecular complex (see Figs. 3a-c, e). We however show in Sect. 3.3 that the highvelocity wing (and possibly part of the low-velocity wing), despite being more than $20 \mathrm{~km} \mathrm{~s}^{-1}$ away from the median velocity

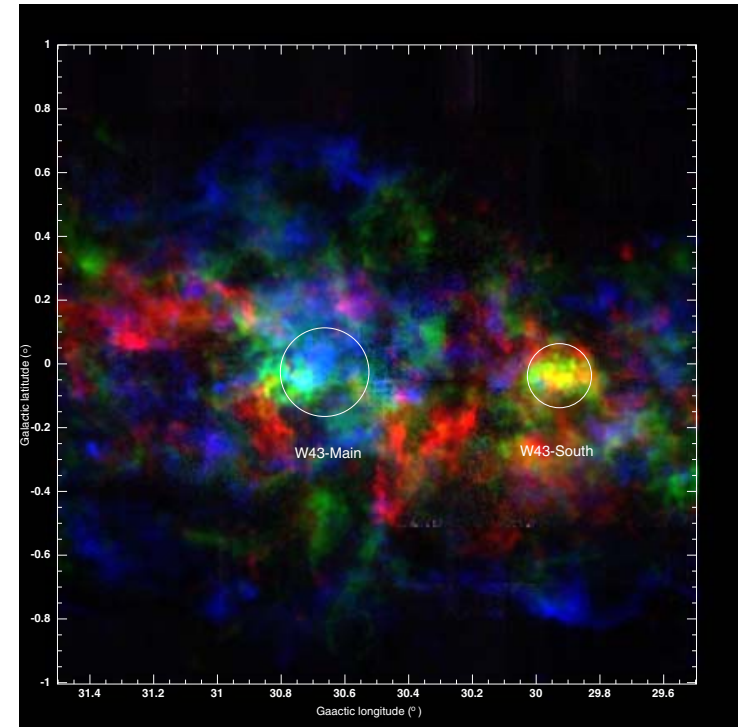

Fig. 5. Composite three-color image of the W43 molecular complex emitting in the ${ }^{13} \mathrm{CO} 1-0$ line: images are integrated over the velocity ranges $80-90 \mathrm{~km} \mathrm{~s}^{-1}$ (blue), $90-100 \mathrm{~km} \mathrm{~s}^{-1}$ (green), and $100-110 \mathrm{~km} \mathrm{~s}^{-1}$ (red). The white circles indicate W43-main and W43south, their areas are used to integrate the spectra of Fig. 2. 


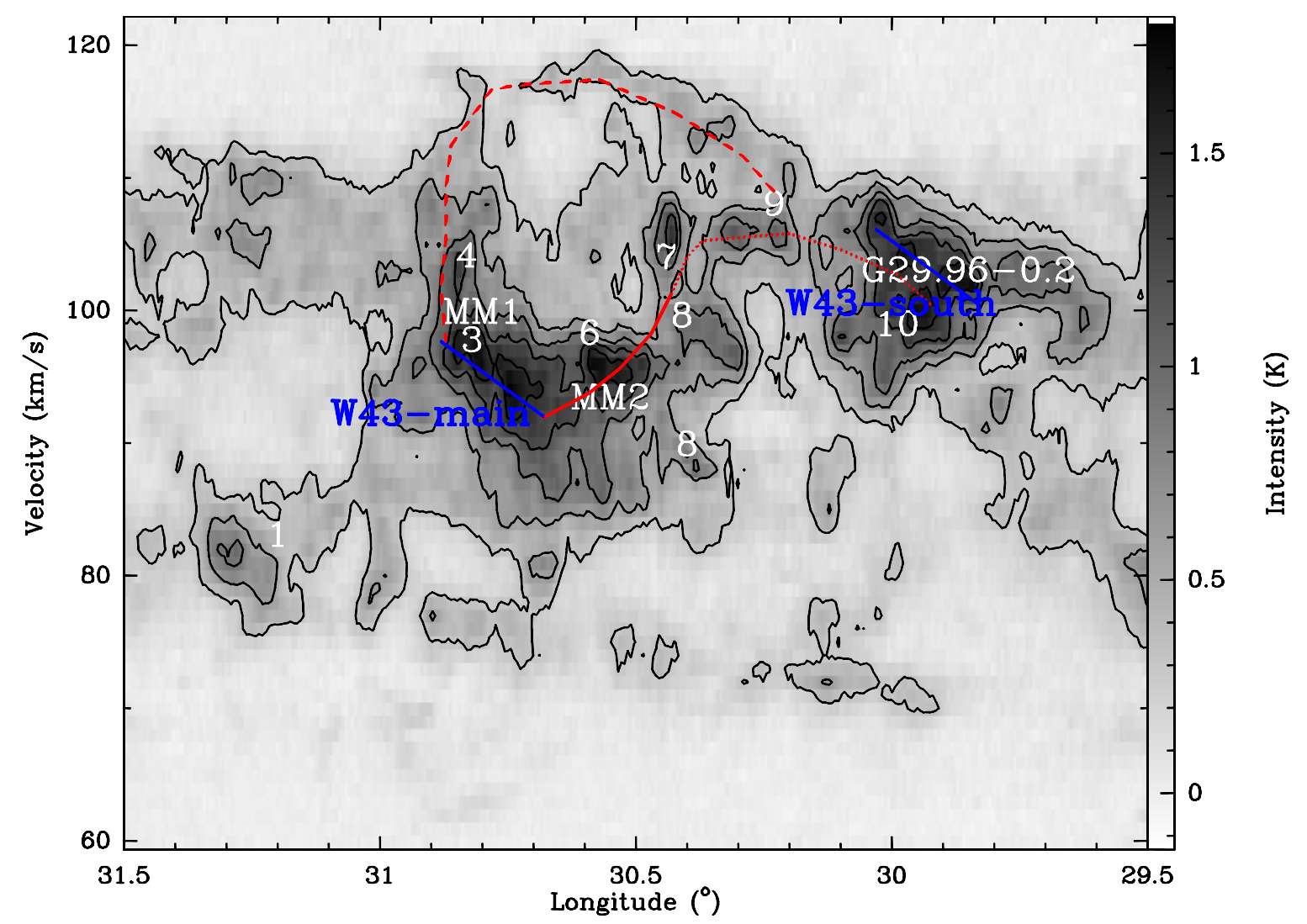

Fig. 6. The position-velocity diagram of the ${ }^{13} \mathrm{CO} 1-0$ line summed over the whole W43 latitude range $\left(-0.5^{\circ}\right.$ to $\left.0.3^{\circ}\right)$ and plotted against its longitude. Contours go from to 0.4 to 2.0 by $0.4 \mathrm{~K}$. The two most prominent structures, W43-main and W43-south, appear to be linked by lower density clouds. The two main bridges identified between W43-main and W43-south (blue bars) are outlined by red curves which are continuous when they correspond to filaments clearly identified by Motte et al. (in prep.) and dashed or dotted for weaker clouds connections. The location of a few $870 \mu \mathrm{m}$ continuum peaks and reference sources are also indicated.

of the W43 molecular complex, consists of lower density clouds linked in the position-velocity space to the densest parts of W43. Assuming that the dust emission seen at $870 \mu \mathrm{m}$ traces the densest parts of the molecular cloud, we verify the coherence of the gas along the velocity axis by comparing ${ }^{13} \mathrm{CO}$ spectra found at the positions of dust peaks. Figure 4 shows the dust emission at $870 \mu \mathrm{m}$ over the whole W43 complex and identifies both two main regions, W43-main (also named G30.8-0.0) and W43south (also referred to as G30.0-0.0), and several tens of smaller clumps. Figure 4 displays the extracted ${ }^{13} \mathrm{CO} 1-0$ spectra at a few bright but randomly selected dust peaks and shows that, except for \#2 and \#6, the spectra have their most prominent peak in the $80-110 \mathrm{~km} \mathrm{~s}^{-1}$ velocity range. None of the spectra shows a secondary peak at $5-15 \mathrm{~km} \mathrm{~s}^{-1}$ and only half of them have a secondary peak at $30-55 \mathrm{~km} \mathrm{~s}^{-1}$. Moreover, LSR velocities measured for these ATLASGAL sources with $\mathrm{NH}_{3}$ observations at Effelsberg all peak in this main velocity range (Wienen et al., in prep.), with a second peak at $30-55 \mathrm{~km} \mathrm{~s}^{-1}$ for \#2 and \#6. The ${ }^{13} \mathrm{CO} 1-0$ lines integrated over the W43-main and W43south regions (as defined in Fig. 4) have also their main velocity component within the $\sim 80-110 \mathrm{~km} \mathrm{~s}^{-1}$ range (see Fig. 2). We therefore confirm that this velocity range is the most relevant for the W43 complex. Using this main velocity range, we delineate in Fig. 3d the W43 molecular complex with a box with longitude from $l=29.6^{\circ}$ to $31.4^{\circ}$ and latitude from $b=-0.5^{\circ}$ to $0.3^{\circ}$. We created a three-color image of the ${ }^{13} \mathrm{CO} 1-0$ emission where blue is $80-90 \mathrm{~km} \mathrm{~s}^{-1}$, green is $90-100 \mathrm{~km} \mathrm{~s}^{-1}$, and red is $100-110 \mathrm{~km} \mathrm{~s}^{-1}$ (see Fig. 5). W43-main and W43-south are mainly seen in the green and red ranges, respectively, in agreement with the shape of their integrated line shown in Fig. 2.

\subsection{W43: a single molecular cloud complex}

Most of the cloud structures seen in high-density tracers such as $870 \mu \mathrm{m}$ continuum are associated with the W43-main and W43south regions. Their spectra peak in velocity ranges close to each other: $\sim 93.8 \mathrm{~km} \mathrm{~s}^{-1}$ for W43-main and $\sim 99.1 \mathrm{~km} \mathrm{~s}^{-1}$ for W43south, suggesting that they could be connected (see Fig. 2).

We used ${ }^{13} \mathrm{CO} 1-0$ and ${ }^{12} \mathrm{CO} 2-1$ data to test this hypothesis by looking for bridges between W43-main and W43-south. The position-velocity diagram of Fig. 6 displays the ${ }^{13} \mathrm{CO}$ emission integrated over the full latitude extent of the W43 complex (from $-0.5^{\circ}$ to $0.3^{\circ}$ ) as a function of the Galactic longitude. This diagram appears to indicate that W43-main and W43-south are connected by medium- to low-density gas with a peak velocity in the spectra increasing from $\sim 94 \mathrm{~km} \mathrm{~s}^{-1}$ to $\sim 105 \mathrm{~km} \mathrm{~s}^{-1}$ and back to $\sim 99 \mathrm{~km} \mathrm{~s}^{-1}$. This main $\mathrm{CO}$ bridge follows a ${ }^{13} \mathrm{CO}$ filament that connects the southern part of W43-main (close to W43-MM2) to source \#6, continues south of source \#8, and ends at source \#9 (see continuous curve in Figs. 4 and 6). The link between source \#9 and source \#10 (equivalent to G29.96-0.2) is more tenuous (see dotted curve in Figs. 4 and 6) but obvious in the ${ }^{12} \mathrm{CO} 2-1$ channel maps, which represents velocities ranging from $99 \mathrm{~km} \mathrm{~s}^{-1}$ to $106 \mathrm{~km} \mathrm{~s}^{-1}$ (see Fig. 7). The second CO bridge follows another filament, which starts at source \#3 (equivalent to W43-MM1), and is connected to source \#4 (see continuous line 


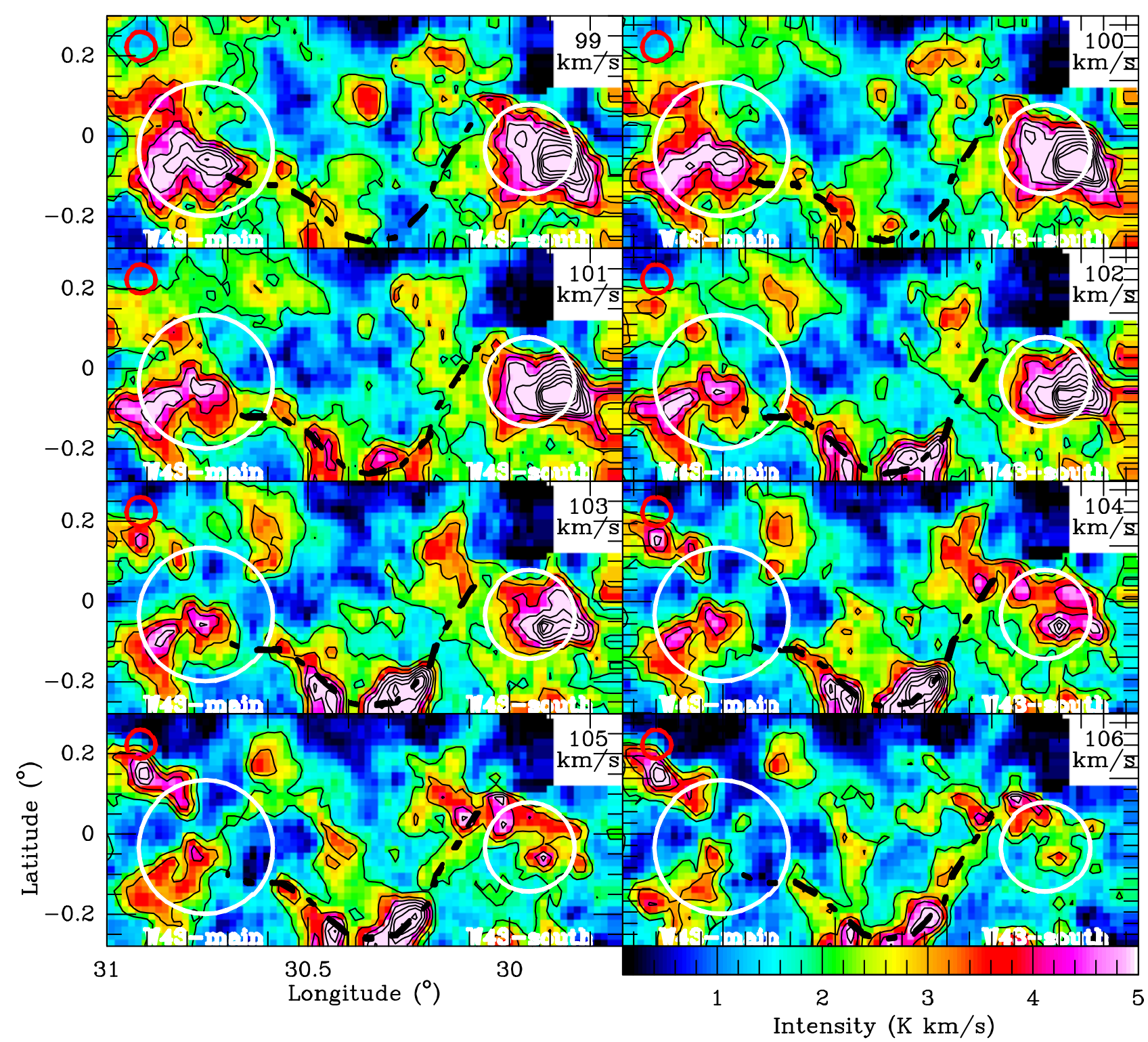

Fig. 7. Velocity maps of the inner part of the W43 molecular complex in the ${ }^{12} \mathrm{CO} 2-1$ emission obtained with KOSMA, integrated over $\sim 1$ km s ${ }^{-1}$ wide channels ranging from $V_{\mathrm{LSR}}=99$ to $106 \mathrm{~km} \mathrm{~s}^{-1}$. The W43-main and W43-south regions are indicated with white circles whose areas are used to integrate the spectra of Fig. 2. The main molecular bridge linking them is outlined by a dashed curve. The HPBW is plotted in the top-left corner.

in Figs. 4 and 6). In Fig. 6, an arc of lower density material then links source \#4 to source \#9 and possibly continues to source \#10, which is G29.96-0.2. We note that this arc of low-density material at velocities $108-120 \mathrm{~km} \mathrm{~s}^{-1}$ corresponds to the velocity ranges that we have excluded from the main velocity range of W43 (Fig. 2) and this stream is shown in Fig. 3e. Figure 6 also displays a line of diffuse clouds lying from $l=31.3^{\circ}$ and $V_{\mathrm{LSR}} \sim 82 \mathrm{~km} \mathrm{~s}^{-1}$ to $l=29.9^{\circ}$ and $V_{\mathrm{LSR}} \sim 71 \mathrm{~km} \mathrm{~s}^{-1}$, respectively, which may or may not be connected to W43-main and W43-south. They correspond to the filamentary clouds, shown in Fig. 3c, which are mostly away from W43-main and W43south. This suggests that most of the low-velocity wing of the ${ }^{13} \mathrm{CO}$ line in Fig. 2 has a lower probability of being related to the W43 molecular complex than its high-velocity wing. A similar high-velocity wing feature is found in the W51 region and the authors speculate it is colliding with the W51 main region (Carpenter \& Sanders 1998; Burton 1970). The fact that W43main and W43-south share the same Hi envelope (see Sect. 3.4) further strengthens the hypothesis that they belong to the same coherent structure in space and velocity.

As we have shown, a broad range of evidence supports the hypothesis that W43-main and W43-south are connected. They are thus probably part of the same molecular cloud structure defined as a GMA. Hereafter, we refer to as "the W43 molecular complex" the complete ensemble of molecular clouds that covers the area $1.8^{\circ} \times 0.8^{\circ}$ around G30.5-0.1 and the velocity range $80-110 \mathrm{~km} \mathrm{~s}^{-1}$. With a $F W H M$ velocity dispersion of $22 \mathrm{~km} \mathrm{~s}^{-1}$, W43 is at the upper range of dispersions determined for Galactic and extragalactic GMCs (5-20 $\mathrm{km} \mathrm{s}^{-1}$, Schneider et al. 2006; Fukui et al. 2009). It arises from the W43 complex consisting of 


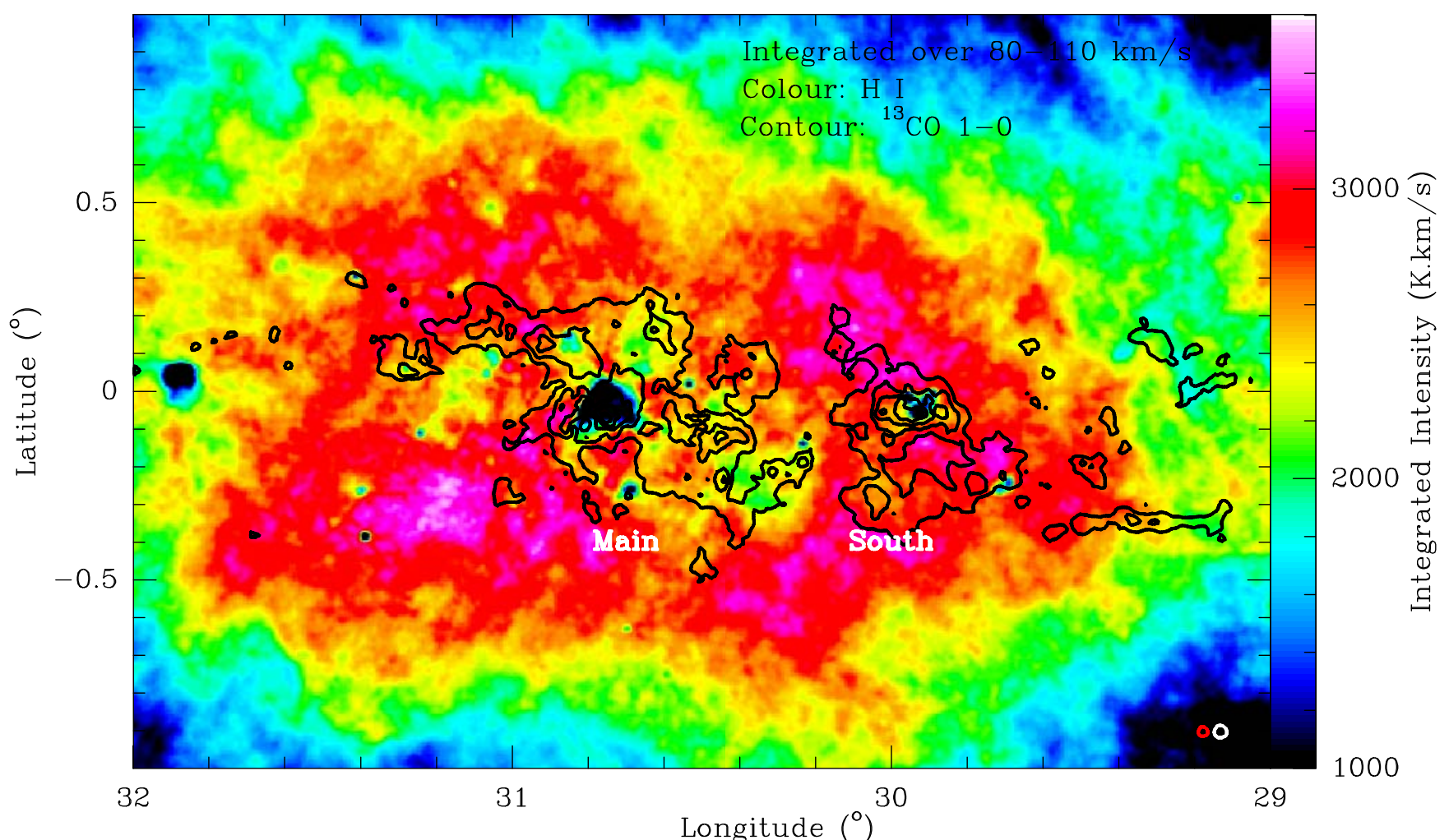

Fig. 8. ${ }^{13} \mathrm{CO} 1-0$ intensity map (contours) overlaid on the Hi line image of the W43 region. Both lines have been integrated over the $80-110 \mathrm{~km} \mathrm{~s}^{-1}$ velocity range. Contours go from to 15 to 75 by 10 in unit of $\mathrm{K} \mathrm{km} \mathrm{s}^{-1}$. The $\mathrm{HI}$ absorption correlates well with the ${ }^{13} \mathrm{CO}$ emission of the W43 molecular cloud complex. The HPBWs are plotted in the bottom-left corner for HI (white) and ${ }^{13} \mathrm{CO} 1-0$ (red).

an association, with quite rich dynamics, of several GMCs that have more typical $\left(\sim 10 \mathrm{~km} \mathrm{~s}^{-1}\right)$ dispersions. Note that the remarkably large velocity dispersion of W43 was also noticeable in Fig. 2a of Dame et al. (1986).

\subsection{W43: surrounded by an atomic gas envelope}

In Fig. 8, the Hi emission associated with the W43 molecular cloud complex, integrated from $80 \mathrm{~km} \mathrm{~s}^{-1}$ to $110 \mathrm{~km} \mathrm{~s}^{-1}$, is compared to that of its ${ }^{13} \mathrm{CO} 1-0$ emission. Above the $\sim 1500 \mathrm{~K} \mathrm{~km} \mathrm{~s}^{-1}$ emission level, the atomic gas surrounds the ${ }^{13} \mathrm{CO}$ molecular complex. The Hi cloud complex of W43 is roughly centered on G30.7-0.05, i.e. $\sim 30 \mathrm{pc}$ away from the molecular complex center as defined by our analysis of the ${ }^{13} \mathrm{CO}$ database (G30.5-0.1, see Sect. 3.3) and assuming a distance of $\sim 6 \mathrm{kpc}$ (see Sect. 4.1). The Hi map shows strong absorption features where ${ }^{13} \mathrm{CO}$ is bright, which is consistent with the trend by Liszt (1995) for W43-main. In general, these absorption features are caused by self-absorption of the $\mathrm{H}_{\mathrm{r}}$ line and/or depletion of $\mathrm{Hi}$ gas that disappears to form molecular gas. The large extent and strength of this self-absorption and/or depletion of the Hi emission is not common in the Galactic plane. Interpreted together, these results suggest that atomic and molecular gas components are closely related in the W43 region. We conclude that there is an atomic gas envelope around the W43 molecular complex, as well as a strong absorption/depletion of atomic gas toward the inner part of the molecular complex.

The position-velocity diagram complied by Elmegreen \& Elmegreen (1987, see their Figs. 1a-c) for the Hi gas in the first quadrant, indicates that there is a $\sim 60-120 \mathrm{~km} \mathrm{~s}^{-1}$ velocity range for the W43 Hi complex, i.e. a larger range than the one defined in Sect. 3.2 for the molecular gas. It also displays the other two velocity components we identifed in ${ }^{13} \mathrm{CO}\left(\sim 5-15 \mathrm{~km} \mathrm{~s}^{-1}\right.$ and $\sim 30-55 \mathrm{~km} \mathrm{~s}^{-1}$, see Fig. 2).

\section{Discussion: defining a new and extreme molecular complex}

\subsection{W43: the closest molecular cloud of the Galactic bar}

W43 appears as a coherent complex in both space and velocity and spans the ranges $l=29.6^{\circ}$ to $31.4^{\circ}, b=-0.5^{\circ}$ to $0.3^{\circ}$, and $V_{\mathrm{LSR}}=80-110 \mathrm{~km} \mathrm{~s}^{-1}$ (see Sect. 3.3). We used a standard model for the Galactic rotation (Brand \& Blitz 1993), with a Galactocentric radius of the Sun of $8.5 \mathrm{kpc}$ to derive the kinematic distance of the W43 molecular cloud complex. This model (Reid et al. 2009) assumes that the source at $V_{\mathrm{LSR}}$ is only given by the differential rotation in the Galaxy and leads, for sources in the inner Galaxy, to one near and one far kinematic distance. With a median longitude of $l \sim 30.5^{\circ}$ and a median radial velocity of $V_{\mathrm{LSR}} \sim 96 \mathrm{~km} \mathrm{~s}^{-1}$, we calculated for W43 a near distance of $\sim 5.9 \mathrm{kpc}$ and a far distance of $\sim 8.7 \mathrm{kpc}$. To resolve the kinematic distance ambiguity, one tries to estimate whether most of the cloud material lies in the foreground or background of the source of interest, thus favors, respectively, its far or near distance. Among the most generally used methods are Hi line measurements of Hir regions, Hi self-absorption features against clouds, and the distribution of near-infrared extinction along the line of sight (see e.g. Anderson \& Bania 2009; Roman-Duval et al. 2009; Schuller et al. 2009). For sources in W43, most 


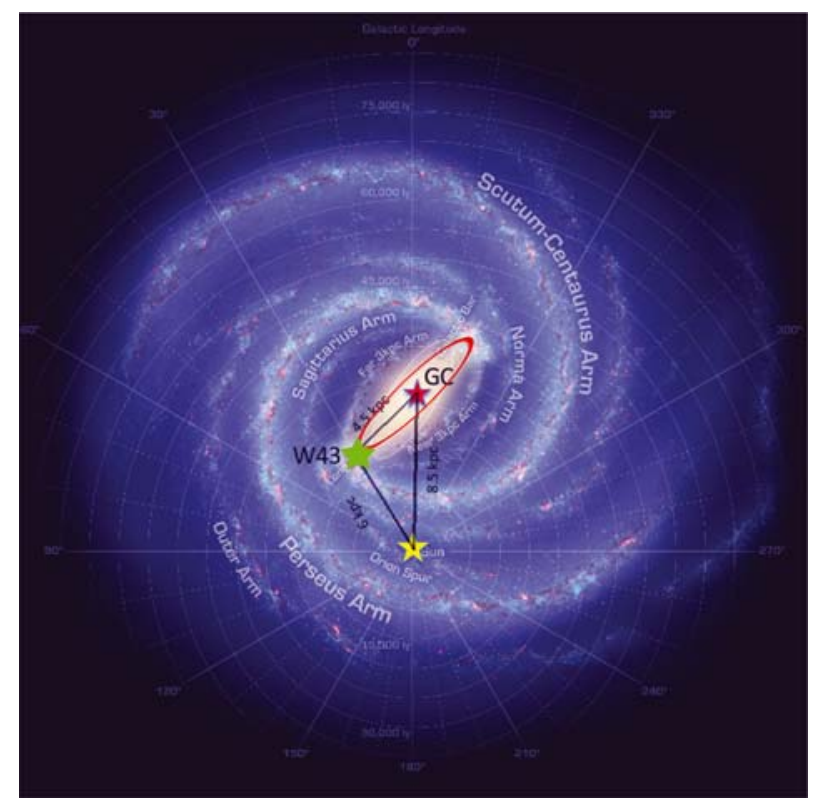

Fig. 9. Artist view of the Galaxy seen face-on with the "long bar" outlined by a red ellipse (Churchwell et al. 2009). W43 is located at the expected transition zone between the bar-dominated region $\left(R_{\mathrm{GC}}<5 \mathrm{kpc}\right)$ and the normal Galactic disk.

authors have argued for the near distance: Anderson \& Bania (2009) for W43-main on the basis of Hi self-absorption, Pratap et al. (1999) using both near-infrared extinction and formaldehyde absorption toward the UCHII region G29.96-0.02 in W43south, Russeil et al. (2011) using various techniques on tens of sources of the W43 region. However, Pandian et al. (2008) using Hi self-absorption toward several HII regions of W43-main favored the far distance. Others simply assumed the average of the near and far distances. Nevertheless, the large-scale absorption of $\mathrm{HI}$, which we investigate in more detail in a forthcoming paper (Motte et al., in prep.), also supports the near-distance of W43.

The structure of the Milky Way remains a matter of debate but in short consists of a disk with spiral arms, a halo, a triaxial bulge, and probably a long bar. Numerical simulations of its gas dynamics assuming a triaxial bulge (a boxy inner structure sometimes called the traditional bar) and without a long bar would place W43 in the transition region from the "lateral arms" and the Scutum-Centaurus arm (e.g. Rodriguez-Fernandez \& Combes 2008). Gas crowding in this region can certainly give rise to the cloud-cloud collisions that may be the origin to the extreme properties of the W43 complex (Rodriguez-Fernandez priv. com.). Observational studies have found evidence of a long in-plane Galactic bar (Hammersley et al. 2000; Benjamin et al. 2005; López-Corredoira et al. 2007) that has a radius of $4.4 \mathrm{kpc}$ and is oriented about $44^{\circ}$ to the $\sim 8.5 \mathrm{kpc}$ Sun-Galactic center line. From the sketch of this Galactic (long) bar given in Fig. 9, we measure a distance from the Sun to the near-tip of the bar of $\sim 6 \mathrm{kpc}$. The tip of the bar is the place where the transition from circular to elliptical orbits in the spiral arm and bar potentials could easily drive high-velocity streams that can collide. The gas response to this bar has never been modeled but would certainly perturb the gas dynamics at the position of W43, and help explain the exceptional characteristics of W43, which is a very massive, highly concentrated molecular complex that actively forms (massive) stars (see Table 2 and Sects. 4.24.5). Additional support for our hypothesis is that extragalactic studies often observe molecular clouds and star-forming regions with extreme properties at the ends of galactic bars (e.g. M 83, NGC 1300, see Martin \& Friedli 1997 and references therein). We also note that the determination of the distance from the kinematic distance has limited accuracy because the true rotation pattern of our Galaxy, especially at the connection point of the Galactic arms and bar, may deviate significantly from the theoretical assumptions of axially symmetric and circular orbits (Russeil 2003; Reid et al. 2009). A deviation of $\sim 10 \mathrm{~km} \mathrm{~s}^{-1}$ due to streaming motions is typical in Galactic arms and would give kinematic distances of $5.9_{-0.7}^{+1.2} \mathrm{kpc}$ and $8.7_{-1.3}^{+0.6} \mathrm{kpc}$. This velocity deviation is smaller than the velocity dispersion measured in molecular complexes such as W43 and Cygnus X (see Table 2). Our study therefore suggests that the W43 molecular complex could be located at $\sim 6 \mathrm{kpc}$ from the Sun and at the connecting point of the Scutum-Centaurus arm and the Galactic bar. The stellar distance of clusters or the parallax of masers excited by HII regions associated with the W43 molecular cloud could help us to check this assumption. Until now, the very few attempts to apply the complementary photo-spectrometric method to the WR-OB cluster of W43-main and the two OB stars within G29.96-0.02 have not given convincing results $(d \sim 4.3 \mathrm{kpc}$, Pratap et al. 1999), probably because the stars are extremely young and highly extinct. It is unfortunately not yet possible to measure the parallax distance of W43 masers since they are rather weak.

\subsection{The total mass of the W43 molecular cloud complex}

We determined the total $\mathrm{H}_{2}$ mass of the W43 molecular complex from the ${ }^{12} \mathrm{CO} 1-0$ data of the CfA survey (Dame et al. 2001). We measured the total intensity of ${ }^{12} \mathrm{CO} 1-0$ over the full area of the W43 complex $\left(A=1.8^{\circ} \times 0.8^{\circ}\right)$ and the velocity range of $80-110 \mathrm{~km} \mathrm{~s}^{-1}$. We then calculated the total gas mass using the conversion factor $N_{\mathrm{H} 2}=2.75 \times 10^{20} \times I\left({ }^{12} \mathrm{CO}\right)$ (Bloemen et al. 1986) and the equation

$$
M_{\mathrm{total}}=N_{\mathrm{H} 2} \times A \times m_{\mathrm{H} 2}=7.1 \times 10^{6} M_{\odot} \times\left(\frac{d}{6 \mathrm{kpc}}\right)^{2} .
$$

The mass estimated with this empirical equation is rather uncertain since some clouds may still lie along the same line of sight, which are unrelated to W43, but it gives an upper limit to the total mass of W43. The computed mass is in the range of values one finds for large molecular cloud structures such as complexes and GMAs (e.g. Kuno et al. 1995). We also calculated the molecular mass of dense clouds in W43 from the ${ }^{13} \mathrm{CO} 1-0$ and ${ }^{12} \mathrm{CO} 2-1$ data. We used the generally adopted scheme, which assumes a uniform medium (filling factor of 1) and that the emission is optically thick in ${ }^{12} \mathrm{CO}$ and optically thin in ${ }^{13}$ CO (e.g. Rohlfs \& Wilson 2000; Schneider et al. 2006). The excitation temperature is first measured from the ${ }^{12} \mathrm{CO} 2-1$ main beam temperature and assumed to apply to the ${ }^{13} \mathrm{CO} 1-0$ transition. We determined $T_{\mathrm{ex}}$ for each pixel of the ${ }^{12} \mathrm{CO}$ map and showed that it ranges from 5 to $18 \mathrm{~K}$ with a mean value of $\sim 10 \mathrm{~K}$. Under the LTE assumption, the column density of ${ }^{13} \mathrm{CO}$ can then be estimated from its intensity with the equation $N\left({ }^{13} \mathrm{CO}\right)=\frac{3.0 \times 10^{4} \mathrm{~cm}^{-2}}{1-\left(\mathrm{e}^{-5.3} \mathrm{~K} / T_{e x}\right)} \int T_{\mathrm{MB}}\left({ }^{13} \mathrm{CO}\right) \mathrm{d} V$. The total column density of ${ }^{13} \mathrm{CO}$ is finally converted into $\mathrm{H}_{2}$ column density by multiplying by a factor of $4.7 \times 10^{5}$ (Rohlfs \& Wilson 2000). We assumed an excitation temperature of $\sim 10 \mathrm{~K}$ for each point and 
estimated the mass from the ${ }^{13} \mathrm{CO}$ intensity integrated over the full extent of the W43 complex

$M_{\text {clouds }} \sim 4.2 \times 10^{6} M_{\odot} \times\left(\frac{d}{6 \mathrm{kpc}}\right)^{2}$.

The difference between $M_{\text {total }}$ and $M_{\text {clouds }}$ illustrates the uncertainty inherent in the mass estimation of $\mathrm{CO}$ clouds. These values are in good agreement with the virial masses measured by Mooney et al. (1995) for the two main clouds W43-main and W43-south and summing up to $\sim 4 \times 10^{6} M_{\odot}$. We note that this $M_{\text {clouds }}$ value is slightly underestimated since some of the ${ }^{13} \mathrm{CO}$ clouds are optically thick. An estimate of the virial mass of W43 was performed using the Gaussian fit to the ${ }^{13} \mathrm{CO}$ line shown in Fig. $2\left(\Delta V \sim 22 \mathrm{~km} \mathrm{~s}^{-1}\right.$ and thus $\sigma=\sqrt{3 /(8 \ln 2)} \times$ $\Delta V \sim 16 \mathrm{~km} \mathrm{~s}^{-1}$ ) and the diameter given in Table 2 is equivalent to $R \sim 70 \mathrm{pc}$

$M_{\mathrm{vir}}=\frac{5 \sigma^{2} \times R}{G}=2.1 \times 10^{7} M_{\odot} \times \frac{d}{6 \mathrm{kpc}}$.

The ratio $M_{\text {total }} / M_{\text {vir }} \sim 0.35$ suggests that the entire W43 molecular complex is probably in gravitational virial equilibrium (Pound \& Blitz 1993). If we only consider the molecular clouds securely associated with W43-main (from $l=30.2^{\circ}$ to $31^{\circ}$ ), we estimate $M_{\text {total }}=5.0 \times 10^{6} M_{\odot}, M_{\text {clouds }}=3.0 \times 10^{6} M_{\odot}$ and $M_{\text {vir }}=1.0 \times 10^{7} M_{\odot}$ leading to $M_{\text {total }} / M_{\text {vir }} \sim 0.5$. These results show that both the W43-main complex and the entire W43 GMA should be in gravitational virial equilibrium, like most of the observed molecular clouds or complexes (e.g. Heyer et al. 2001). They also support the idea that the W43 GMA is indeed a single molecular cloud entity bounded by self-gravity and that its individual molecular clouds could even be collapsing.

\subsection{The mass of compact clumps in W43}

The ATLASGAL image of W43 (see Fig. 4) enables us to probe the distribution of relatively large (up to $\sim 10 \mathrm{pc}$ ) cloud structures by analyzing their dust continuum emission at $870 \mu \mathrm{m}$. To trace the mass of clumps with size $<5 \mathrm{pc}$, we filtered out the emission on large scales by applying the wavelet transform technique of Starck \& Murtagh (2006) as used by Motte et al. (2007). If we assume that the $870 \mu \mathrm{m}$ emission is optically thin and consists mostly of thermal dust emission, the total gas + dust mass of the W43 clumps is simply proportional to its total flux, $S_{870}$, integrated over the $1.8^{\circ} \times 0.8^{\circ}$ area defined in Sect. 3 . We used an equation similar to Eq. (1) of Motte et al. (2003) and estimated a mass for W43 clumps of

$$
\begin{aligned}
M_{\text {clumps }} & =8.4 \times 10^{5} M_{\odot}\left(\frac{e^{\frac{16.58}{T_{\text {dust }}}}-1}{e^{\frac{16.58}{20 \mathrm{~K}}}-1}\right) \\
& \times\left(\frac{\kappa 870 \mu \mathrm{m}}{0.015 \mathrm{~cm}^{2} \mathrm{~g}^{-1}}\right)^{-1}\left(\frac{d}{6 \mathrm{kpc}}\right)^{2}
\end{aligned}
$$

We assumed a dust temperature of $20 \mathrm{~K}$, which is typical of compact cloud structures in high-mass star-forming regions and in good agreement with the $T_{\text {dust }}$ values measured by Motte et al. (2003) for a few W43 clumps and by Molinari et al. (2010) for the W43 complex. We also assumed a dust emissivity of $\kappa 870 \mu \mathrm{m}=0.015 \mathrm{~cm}^{2} \mathrm{~g}^{-1}$, which is intermediate between that recommended for protostellar envelopes and prestellar cores (Ossenkopf \& Henning 1994). The mass estimate of each clump is uncertain by a factor of $\sim 2$ because of our poor knowledge of its dust temperature and emissivity but over the complete cloud the average uncertainty should be lower. Since dust emissivity may increase with the metallicity of the medium, thus be larger at a Galactocentric distance of $4 \mathrm{kpc}$ (W43) than at $8 \mathrm{kpc}$ (solar neighborhood), the mass given above could be overestimated by up to a factor of 1.4 (see Mooney et al. 1995 and references therein). We note that taking the metallicity into account would also decrease the mass estimates of $M_{\text {total }}$ and $M_{\text {clouds }}$.

\subsection{W43 compared to other prominent cloud complexes}

In the Galactic plane, the most prominent cloud complexes are relatively nearby (at 1-3 kpc from the Sun), such as M16-M17, NGC 6334-6357, Cygnus X, NGC 7538, W48, or W3. Further away, one has found extreme star-forming regions, such as W43, W49, or W51 and of course the central molecular zone (CMZ). A complete census and characterization of massive molecular complexes in our Galaxy is underway in the framework of the ATLASGAL and Hi-GAL surveys (see first study by Schuller et al. 2009 and Molinari et al. 2010). Given our present view, we however believe that W43 will remain one of the most extreme molecular complexes in the Milky Way that is also relatively near to the Sun.

The W43 molecular complex is located more than three times further away from the Sun than Cygnus $\mathrm{X}$ but has a similar linear size. The total mass of $\mathrm{H}_{2}$ gas in W43 is very high, even slightly higher than in the Cygnus $\mathrm{X}$ molecular complex (see Table 2), which is already among the most massive complexes of our Galaxy. This trend is more significant when we compare, in W43 and Cygnus X, the mass accumulated within clouds identified in ${ }^{13} \mathrm{CO}$ and clumps ( $<5 \mathrm{pc}$ cloud structures): it is $\sim 5$ and almost 15 times larger in W43. Interestingly enough, the clumps-to-total mass ratio found in W43 is $\sim 10 \%$, definitively much larger than that found in Cygnus X $(\sim 1 \%$, Motte et al. 2007). About $20 \%$ of the ${ }^{13} \mathrm{CO}$ cloud mass in W43 is located in high-density clumps, which is a similar fraction to that observed for extreme star-forming regions, such as W49, W51, and the $\mathrm{CMZ}$, despite the ${ }^{13} \mathrm{CO}$ cloud mass being measured differently. Thus, W43 is a massive molecular complex $\left(7.1 \times 10^{6} M_{\odot}\right.$ within a linear diameter of $\left.\sim 140 \mathrm{pc}\right)$, which is exceptional in terms of the concentration of its gas into ${ }^{13} \mathrm{CO}$ cloud $\left(4.2 \times 10^{6} M_{\odot}\right)$ and compact cloud structures $\left(8.4 \times 10^{5} M_{\odot}\right.$ within $5 \mathrm{pc}$ dense clumps). The velocity dispersion determined by a Gaussian fit $\left(\Delta V \sim 22 \mathrm{~km} \mathrm{~s}^{-1}\right)$ for W43 is so large that most of the previous studies separated the region into two complexes, located over the same range of Galactic coordinates but at different velocities and with more ordinary internal motions (at $V_{\mathrm{LSR}} \sim 80 \mathrm{~km} \mathrm{~s}^{-1}$ and $\sim 100 \mathrm{~km} \mathrm{~s}^{-1}$ with $\Delta V \sim 15 \mathrm{~km} \mathrm{~s}^{-1}$, e.g. Dame et al. 1986). We have shown that W43 can be defined as a single and coherent molecular complex (see Sects. 3.3-3.4), which consists of several velocity streams/density filaments associated with the densest parts of W43. It is difficult to compare the velocity dispersions of W43 to other cloud complexes listed in Table 2 since the Gaussian fits are generally not computed in the literature. However, considering the velocity spans of those clouds, the total velocity dispersion of W43 seems significantly larger than that of Cygnus X, W49, and W51.

\subsection{The $\mathrm{H}_{\mathrm{I}}$ envelope of $\mathrm{W} 43$ and the W43 $\left(\mathrm{HI}+\mathrm{H}_{2}\right)$ cloud}

The Hi gas envelopes the W43 molecular cloud, therefore covers a larger area and possibly a larger velocity range than the ${ }^{13} \mathrm{CO}$ cloud. From Fig. 8, we estimate that it covers the whole 
Table 2. Global characteristics of the W43 molecular and star-forming complex in comparison with other star-forming complexes.

\begin{tabular}{|c|c|c|c|c|c|c|c|}
\hline Region & $\begin{array}{l}\text { Assumed } \\
\text { distance } \\
{[\mathrm{kpc}]} \\
\end{array}$ & $\begin{array}{l}\text { Equivalent diameter } \\
\text { from }{ }^{13} \mathrm{CO} \\
{[\mathrm{pc}]}\end{array}$ & $\begin{array}{l}\text { Velocity range } \\
\text { from }{ }^{13} \mathrm{CO} \\
{\left[\mathrm{km} \mathrm{s}^{-1}\right]}\end{array}$ & $\begin{array}{l}\text { Total } \mathrm{H}_{2} \text { Mass } \\
\text { from }{ }^{12} \mathrm{CO} \\
{\left[M_{\odot}\right]}\end{array}$ & $\begin{array}{l}\text { Clouds mass } \\
\text { from }{ }^{13} \mathrm{CO} \\
{\left[M_{\odot}\right]}\end{array}$ & $\begin{array}{l}\text { Dense clumps mass } \\
\text { from submm }{ }^{a} \\
{\left[M_{\odot}, \%\right]}\end{array}$ & $\begin{array}{l}8 \mu \mathrm{m} \text { luminosity } \\
\text { from } 8 \mu \mathrm{m}^{b} \\
{\left[L_{\odot}\right]}\end{array}$ \\
\hline W43 & 6.0 & $\sim 140$ & 80 to 110 & $7.1 \times 10^{6}$ & $4.2 \times 10^{6}$ & $8.4 \times 10^{5}, 12 \%$ & $1.60 \times 10^{7}$ \\
\hline Cygnus X & $1.7^{c}$ & $\sim 160^{c}$ & -10 to $20^{c}$ & $5.0 \times 10^{6 c}$ & $8.0 \times 10^{5 c}$ & $6.0 \times 10^{4}, 1 \%^{d}$ & $6.57 \times 10^{6 e}$ \\
\hline W51 & 7.0 & $\sim 100^{f}$ & 56 to $65^{f}$ & $\sim 1.2 \times 10^{6 f}$ & $\ldots$ & $5.5 \times 10^{5}$ & $\ldots$ \\
\hline CMZ & 8.5 & $\sim 350^{g}$ & -225 to $225^{g}$ & $\sim 3.0 \times 10^{8 g}$ & $\sim 2.0 \times 10^{7 g}$ & $4.0 \times 10^{6}$ & $\ldots$ \\
\hline W49 & $11.4^{h}$ & $\sim 45^{h}$ & -5 to $25^{h}$ & $\ldots$ & $\sim 5.0 \times 10^{5 h}$ & $2.0 \times 10^{5}$ & $\ldots$ \\
\hline
\end{tabular}

Notes. ${ }^{(a)}$ The dense clumps mass is calculated from the ATLASGAL $870 \mu \mathrm{m}$ images and MAMBO2 $1.3 \mathrm{~mm}$ for Cygnus X, from which $\geqslant 5$ pc cloud structures have been filtered (more precisely, $5.6 \mathrm{pc}$ for W43, $5 \mathrm{pc}$ for Cygnus X, $6.5 \mathrm{pc}$ for W51, 4.0 pc for CMZ, and 5.3 pc for W49), and using Eq. (4). The percentage of dense clumps formed out of the total $\mathrm{H}_{2}$ gas (taken from column 5) is estimated for the regions where the total $\mathrm{H}_{2}$ mass is more reliable; (b) The $8 \mu \mathrm{m}$ luminosity is measured from band 4 of Spitzer, which has a bandwidth of $2.93 \mu \mathrm{m}$ centered at $7.91 \mu \mathrm{m}$; ${ }^{(c)}$ From Schneider et al. (2006); ${ }^{(d)}$ From Motte et al. (2007) at 1.2mm; ${ }^{(e)}$ From Hora et al. (2009); ${ }^{(f)}$ From Carpenter \& Sanders (1998); ${ }^{(g)}$ From Dahmen et al. (1998) using $\mathrm{C}^{18} \mathrm{O} ;{ }^{(h)}$ From Simon et al. (2001).

image, i.e. $3^{\circ} \times 2^{\circ}$ or a mean diameter of $\sim 290 \mathrm{pc}$ and the position-velocity diagram of Elmegreen \& Elmegreen (1987) gives a velocity range for the $\mathrm{HI}$ gas that could be twice as large as that of molecular complexes, at up to $\sim 60-120 \mathrm{~km} \mathrm{~s}^{-1}$. In contrast to ${ }^{13} \mathrm{CO}$, the confusion along the line of sight is very large at a longitude close to $l \sim 30^{\circ}$ since the intensity measured on the total velocity range of the VGPS $\left(-120\right.$ to $\left.170 \mathrm{~km} \mathrm{~s}^{-1}\right)$ is $\sim 4.4$ times larger than the one measured by limiting the velocity range to $80-110 \mathrm{~km} \mathrm{~s}^{-1}$. For a meaningful comparison of the atomic and molecular gas, we consider only $\mathrm{Hi}$ emission within that range.

We determined the column density of Hi assuming that the W43 $\mathrm{HI}$ cloud is uniform and optically thin and using its integrated intensity over the velocity range $80-110 \mathrm{~km} \mathrm{~s}^{-1}$ in the equation $N_{H I}=1.82 \times 10^{18} \mathrm{~cm}^{-2} \int T_{\mathrm{MB}}(\mathrm{HI}) \mathrm{d} V($ Spitzer 1978). The column density map is then integrated over the $3^{\circ} \times 2^{\circ}$ area and gives $M_{\mathrm{HI}} \sim 3.2 \times 10^{6} M_{\odot}$, which agrees with the value calculated by Elmegreen \& Elmegreen (1987) under the same assumptions. Approximately $20 \%$ of the Hi image of Fig. 8 displays absorption features. If they correspond to self-absorption, its high-level (by factors of $\sim 1.5$ on average and up to $>3$ in W43-main) could lead us to underestimate the total Hi mass by up to $50 \%$, i.e. $\sim 6.4 \times 10^{6} M_{\odot}$. We therefore estimate the Hi gas mass to be

$M_{\mathrm{HI}} \sim 3.2-6.4 \times 10^{6} M_{\odot} \times\left(\frac{d}{6 \mathrm{kpc}}\right)^{2}$.

The estimates of Sects. 4.2 and 4.3 give a total mass for the $\mathrm{HI}+\mathrm{H}_{2}$ cloud of $\sim 1-1.2 \times 10^{7} M_{\odot}$. The molecular mass fraction of W43 is roughly estimated to be $\sim 65 \%$. This agrees with the tendency to have a large molecular mass fraction $(\sim 70 \%)$ in the Galactic center and a small one $(\sim 5 \%)$ in the outer Galaxy (Elmegreen \& Elmegreen 1987). This may suggest a stronger link between the $\mathrm{HI}$ and $\mathrm{H}_{2}$ gas components in W43 as well as a faster formation of molecular clouds than in the outer Galaxy. The special location of W43 may promote its ability to accumulate Hi gas from different structures (arm, halo, bar) and become a large potential well that forms molecular clouds very fast and efficiently. When massive stars are formed, the molecular gas is photo-dissociated back to become Hi again. These two sources of Hi create the envelope-like emission morphology seen in Fig. 8.

\subsection{W43: an exceptional star-forming region}

The W43 molecular complex is understood to be associated with rich clusters of massive stars as well as more recent episodes of star-formation, because it contains high-mass protostars and CHII regions. It is well-known that W43-main contains a giant $\mathrm{H}$ II region emitting $10^{51}$ Lyman continuum photons $\mathrm{s}^{-1}$ and having a far-infrared continuum luminosity of $\sim 3.5 \times 10^{6} L_{\odot}$ (Smith et al. 1978). It hosts an ionizing source that was discovered in near-infrared images by Lester et al. (1985) and confirmed by Blum et al. (1999) as a cluster of WR and OB main-sequence stars. The high luminosity and ionizing flux of the W43-main star-forming region is comparable to that of the very massive star cluster NGC 3603 or M17, which suggests that the central cluster in W43 contains a large number of as yet undetected massive stars. The submillimeter continuum and $\mathrm{HCO}^{+}(3-2)$ survey of Motte et al. (2003) revealed $\sim 50$ massive (20-3600 $M_{\odot}$ ) clumps probably forming high-mass stars thus suggesting that the molecular cloud is undergoing a second remarkably efficient episode of high-mass star-formation ( $S F E$ $25 \% / 10^{6} \mathrm{yr}$ ). The W43-south region also hosts the well-studied CHII region/hot molecular core G29.96-0.02, which has a luminosity of $\sim 4.4 \times 10^{5} L_{\odot}$ (Wood \& Churchwell 1989; Cesaroni et al. 1994; Cesaroni et al. 1998). This CHII is excited by an O5O8 star (Watson \& Hanson 1997) and is associated with an embedded cluster containing massive stars located at its rim (Pratap et al. 1999).

The $8 \mu \mathrm{m}$ flux integrated over the W43 molecular complex gives a rough estimate of the recent (high-mass) star-formation activity. Indeed, the Spitzer/IRAC band 4 is dominated by the emission of PAH particles, which trace the current UV field and thus the number of young OB stars and HII regions (e.g. Peeters et al. 2004). We calculated the $8 \mu$ m luminosity of W43 from the $8 \mu \mathrm{m}$ flux to allow comparison with other star-forming regions (see Table 2 and Sect. 3.1) based on the equation

$$
L=2.74 \times 10^{-10} \times \frac{F}{[\mathrm{MJy} / \mathrm{sr}]} \times \frac{A}{\left[{ }^{\prime 2}\right]} \times \frac{d^{2}}{\left[\mathrm{pc}^{2}\right]}\left[L_{\odot}\right] .
$$

Since W43 lies in the inner part of the Galactic plane, its $8 \mu \mathrm{m}$ luminosity should be corrected for emission arising from other regions along the same line of sight. We estimated this background and foreground emission in the W43 region by measuring the $8 \mu \mathrm{m}$ fluxes just around the molecular complex where there is no dominant emission from compact sources. The contaminating emission accounts for about $1 / 3$ of the total flux. Table 2 reports the $8 \mu \mathrm{m}$ luminosity corrected for the infrared background+foreground for W43 and the uncorrected $8 \mu \mathrm{m}$ luminosity for Cygnus X. We show that the $8 \mu \mathrm{m}$ luminosity and thus the star-formation activity of W43 could be almost 3.5 times higher 
than that of Cygnus $X$. This is remarkable since Cygnus $\mathrm{X}$ is recognized to be one of the most active star-forming complexes of our Galaxy (Hora et al. 2009). We estimate the "current" starformation rate $(S F R)$ of W43 using the $8 \mu \mathrm{m}$ luminosity measured here and equations derived by $\mathrm{Wu}$ et al. (2005) from the Spitzer imaging of galaxies and the SFR formula of Kennicutt (1998)

$$
S_{F R} \mu \mathrm{m}=\frac{v L_{v}[8 \mu \mathrm{m}]}{1.57 \times 10^{9} L_{\odot}} \sim 0.01 M_{\odot} \mathrm{yr}^{-1} \times\left(\frac{d}{6 \mathrm{kpc}}\right)^{2} .
$$

The $8 \mu \mathrm{m} S F R$ traces the young and already-formed stars, but it does not represent the $S F R$ in the future. The latter can be estimated from the molecular gas content in W43. Assuming that the W43 molecular complex, with a mass of $\sim 7.1 \times 10^{6} M_{\odot}$, is forming stars with a $S F E$ of a few percent $(S F E \sim 1 \%-3 \%$, Silk 1997), we expect it to form $\sim 1.4 \times 10^{5} M_{\odot}$ of stars. With a typical lifetime of high-mass star-forming regions of $\sim 1-3 \times$ $10^{6} \mathrm{yr}$ (Roman-Duval et al. 2009), in agreement with scenarios of cloud formation from colliding flows (Heitsch \& Hartmann 2008; Hennebelle et al. 2008; Vázquez-Semadeni et al. 2007), this estimate would yield a "future" SFR of

$$
S F R_{\mathrm{CO}}=0.05-0.14 M_{\odot} \mathrm{yr}^{-1} \times\left(\frac{M_{\text {total }}}{7.1 \times 10^{6} M_{\odot}}\right) \times\left(\frac{S F E}{2 \%}\right) .
$$

The lower value is five times larger than the "current" SFR obtained from the $8 \mu \mathrm{m}$ luminosity. The upper value is even larger (14 times larger) and agrees with the estimate made by Motte et al. (2003) for W43-main only. This result confirms that W43 will be very efficient in forming stars, $\sim 10$ times more efficient than it has already been in the past, despite the presence of the young $\left(\sim 10^{6} \mathrm{yr}\right)$ cluster of WR-OB stars in W43-main. We may be witnessing the formation of new starburst clusters in the W43 region. With a $S F R$ of $\sim 0.1 M_{\odot} \mathrm{yr}^{-1}$ and a lifetime of $\sim 2 \times 10^{6} \mathrm{yr}$, we should expect $\sim 2 \times 10^{5} M_{\odot}$ of stars to form in the W43 molecular cloud, among which there should be about $2.4 \times 10^{4} M_{\odot}$ (12\% of total stellar mass) of stars with masses higher than $8 M_{\odot}$ using the initial mass function (IMF) of Kroupa (2001). If all of this mass were to be converted into stars of $8 M_{\odot}$ or $50 M_{\odot}$, the total number of these stars would be $\sim 3000$ or $\sim 500$, respectively.

\section{Conclusions}

In the framework of the ATLASGAL survey of star-formation, we have identified a new molecular cloud complex located around G30.5-0.1, which includes W43-main and W43-south. We used a large database tracing diffuse atomic gas (HI emission at $21 \mathrm{~cm}$ from the VGPS survey), low- to medium-density molecular gas $\left({ }^{12} \mathrm{CO} 1-0\right.$ and ${ }^{13} \mathrm{CO} 1-0$ emission from the CfA and GRS surveys), high-density molecular gas $(870 \mu \mathrm{m}$ continuum emission from the ATLASGAL survey), and starformation activity ( $8 \mu \mathrm{m}$ from the GLIMPSE survey) and obtained KOSMA observations of the ${ }^{12} \mathrm{CO} 2-1,3-2$ line emission. Our main findings can be summarized as follows:

1. From the detailed 3D (space-space-velocity) analysis of the molecular tracers $\left({ }^{13} \mathrm{CO} 1-0\right.$ data cubes of the GRS and ${ }^{12} \mathrm{CO} 2-1,3-2$ data cubes of KOSMA) throughout the region, we identified W43 as a coherent complex of molecular clouds. It covers a spatial extent of $\sim 140 \mathrm{pc}$ and a velocity range of $\sim 22.3 \mathrm{~km} \mathrm{~s}^{-1} F W H M$ and spans the ranges $l=29.6^{\circ}$ to $31.4^{\circ}, b=-0.5^{\circ}$ to $0.3^{\circ}$, and at least $V_{\mathrm{LSR}}=$ $80-110 \mathrm{~km} \mathrm{~s}^{-1}$. These values show that W43 is a large complex of clouds with a wide velocity dispersion.
2. The analysis of the atomic gas data cube from the VGPS indicates that the W43 molecular complex is surrounded by an atomic gas envelope of larger diameter, $\sim 290 \mathrm{pc}$, and the same or larger velocity range, up to $\sim 60 \mathrm{~km} \mathrm{~s}^{-1}$.

3. The distance we estimated for the W43 complex is $\sim 6 \mathrm{kpc}$ from the Sun. It is therefore located it at the meeting point of the Scutum-Centaurus Galactic arm and the bar. This is a very dynamic region of our Galaxy since it coincides with the place where the transition from circular to elliptical orbits in the spiral arm and bar potentials could easily drive highvelocity streams that can collide.

4. We measured the total mass of the W43 molecular complex to be $M_{\text {total }} \sim 7.1 \times 10^{6} M_{\odot}$ and the mass contained in dense $870 \mu \mathrm{m}$ clumps ( $<5 \mathrm{pc}$ dense cloud structures) to be $M_{\text {clumps }} \sim 8.4 \times 10^{5} M_{\odot}$. When compared to Cygnus X and the CMZ, these values imply that W43 is a massive and concentrated molecular cloud complex. These findings agree with W43 being in the region sometimes called the Molecular Ring and known to be particularly rich in terms of molecular clouds and star-formation activity.

5. We estimated the SFR of W43 using 1) the $8 \mu \mathrm{m}$ luminosity measured by Spitzer in W43 and 2) the mass of W43, the classical $S F E$, and the cloud lifetime, inferring an increase from $S F R \sim 0.01 M_{\odot} \mathrm{yr}^{-1} \sim 10^{6} \mathrm{yr}$ ago to $0.1 M_{\odot} \mathrm{yr}^{-1}$ in the near future. We may be witnessing the formation of new starburst clusters in the W43 region. This result generalizes the study of Motte et al. (2003), which was dedicated to W43main only.

6. We compared the global properties (mass, density, dynamic state, and star-formation activity) of the W43 molecular cloud and star-forming complex to those of other prominent regions and have found that it is one of the most extreme molecular complexes of the Milky Way.

7. Located at only $6 \mathrm{kpc}$ from the Sun, W43 is an excellent laboratory to study the star-formation process. The Herschel Key Program Hi-GAL has already observed this region (e.g. Molinari et al. 2010) and a detailed analysis of the starformation content of this molecular cloud is ongoing.

8. With its location, extreme mass/density characteristics, and ab-normal velocity dispersion, W43 is the perfect laboratory to investigate the formation of an extreme complex of starformation in the framework of the scenario of converging flows. A companion paper, Motte et al. (in prep.), provides the first signatures of colliding flows and the basis of future numerical simulations dedicated to W43. A large program with the IRAM $30 \mathrm{~m}$ designed to determine the diagnostics/signatures of colliding flows from high- and low-density $\mathrm{HI} / \mathrm{CO}$ streams to low- and high-density star-formation seeds is ongoing (Motte et al. 2003).

Acknowledgements. We thank Nemesio Rodriguez-Fernandez and Françoise Combes for suggestions that improved the manuscript. We thank Joe Hora for providing his Spitzer images of Cygnus X for comparative measurements, Thomas Dame for providing the ${ }^{12} \mathrm{CO} 1-0$ cubes of his Galactic plane survey and Marion Wienen for providing the $\mathrm{NH}_{3} V_{\mathrm{LSR}}$ prior to publication. Part of this work was supported by the ANR (Agence Nationale pour la Recherche) project "PROBeS", number ANR-08-BLAN-0241. L.B. acknowledges support from CONICYT projects FONDAP 15010003 and Basal PFB-06.

\section{References}

Anderson, L. D., \& Bania, T. M. 2009, ApJ, 690, 706

Bally, J., Anderson, L. D., Battersby, C., et al. 2010, A\&A, 518, L90

Benjamin, R. A., Churchwell, E., Babler, B. L., et al. 2003, PASP, 115, 953

Benjamin, R. A., Churchwell, E., Babler, B. L., et al. 2005, ApJ, 630, L149

Beuther, H., Zhang, Q., Bergin, E. A., et al. 2007, A\&A, 468, 1045 
Bloemen, J. B. G. M., Strong, A. W., Mayer-Hasselwander, H. A., et al. 1986, A\&A, 154, 25

Blum, R. D., Damineli, A., \& Conti, P. S. 1999, AJ, 117, 1392

Brand, J., \& Blitz, L. 1993, A\&A, 275, 67

Burton, W. B. 1970, A\&AS, 2, 291

Carpenter, J. M., \& Sanders, D. B. 1998, AJ, 116, 1856

Cesaroni, R., Churchwell, E., Hofner, P., Walmsley, C. M., \& Kurtz, S. 1994, A\&A, 288, 903

Cesaroni, R., Hofner, P., Walmsley, C. M., \& Churchwell, E. 1998, A\&A, 331, 709

Churchwell, E., Babler, B. L., Meade, M. R., et al. 2009, PASP, 121, 213

Comerón, F., \& Torra, J. 1994, A\&A, 281, 35

Dahmen, G., Huttemeister, S., Wilson, T. L., \& Mauersberger, R. 1998, A\&A, 331,959

Dame, T. M., Elmegreen, B. G., Cohen, R. S., \& Thaddeus, P. 1986, ApJ, 305, 892

Dame, T. M., Hartmann, D., \& Thaddeus, P. 2001, ApJ, 547, 792

Elmegreen, B. G., \& Elmegreen, D. M. 1987, ApJ, 320, 182

Fukui, Y., Kawamura, A., Wong, T., et al. 2009, ApJ, 705, 144

Graf, U. U., Haas, S., Honingh, C. E., et al. 1998, in SPIE Conf. Ser. 3357, ed. T. G. Phillips, 159

Hammersley, P. L., Garzón, F., Mahoney, T. J., López-Corredoira, M., \& Torres, M. A. P. 2000, MNRAS, 317, L45

Heitsch, F., \& Hartmann, L. 2008, ApJ, 689, 290

Hennebelle, P., Banerjee, R., Vázquez-Semadeni, E., Klessen, R. S., \& Audit, E. 2008, A\&A, 486, L43

Heyer, M. H., Carpenter, J. M., \& Snell, R. L. 2001, ApJ, 551, 852

Hora, J. L., Bontemps, S., Megeath, S. T., et al. 2009, in BAAS, 41, 498

Jackson, J. M., Rathborne, J. M., Shah, R. Y., et al. 2006, ApJS, 163, 145

Kennicutt, Jr., R. C. 1998, ApJ, 498, 541

Koda, J., Scoville, N., Sawada, T., et al. 2009, ApJ, 700, L132

Kramer, C., Degiacomi, C. G., Graf, U. U., et al. 1998, in SPIE Conf. Ser. 3357, ed. T. G. Phillips, 711

Kroupa, P. 2001, MNRAS, 322, 231

Kuno, N., Nakai, N., Handa, T., \& Sofue, Y. 1995, PASJ, 47, 745

Lester, D. F., Dinerstein, H. L., Werner, M. W., et al. 1985, ApJ, 296, 565

Liszt, H. S. 1995, AJ, 109, 1204
López-Corredoira, M., Cabrera-Lavers, A., Mahoney, T. J., et al. 2007, AJ, 133, 154

Martin, P., \& Friedli, D. 1997, A\&A, 326, 449

Molinari, S., Swinyard, B., Bally, J., et al. 2010, A\&A, 518, L100

Mooney, T., Sievers, A., Mezger, P. G., et al. 1995, A\&A, 299, 869

Motte, F., Schilke, P., \& Lis, D. C. 2003, ApJ, 582, 277

Motte, F., Bontemps, S., Schilke, P., et al. 2007, A\&A, 476, 1243

Ossenkopf, V., \& Henning, T. 1994, A\&A, 291, 943

Pandian, J. D., Momjian, E., \& Goldsmith, P. F. 2008, A\&A, 486, 191

Peeters, E., Spoon, H. W. W., \& Tielens, A. G. G. M. 2004, ApJ, 613, 986

Pound, M. W., \& Blitz, L. 1993, ApJ, 418, 328

Pratap, P., Megeath, S. T., \& Bergin, E. A. 1999, ApJ, 517, 799

Rand, R. J. 1993, ApJ, 410, 68

Rand, R. J., \& Kulkarni, S. R. 1990, ApJ, 349, L43

Rathborne, J. M., Johnson, A. M., Jackson, J. M., Shah, R. Y., \& Simon, R. 2009, ApJS, 182, 131

Reid, M. J., Menten, K. M., Zheng, X. W., et al. 2009, ApJ, 700, 137

Rodriguez-Fernandez, N. J., \& Combes, F. 2008, A\&A, 489, 115

Rohlfs, K., \& Wilson, T. L. 2000, Tools of radio astronomy (Springer)

Roman-Duval, J., Jackson, J. M., Heyer, M., et al. 2009, ApJ, 699, 1153

Russeil, D. 2003, A\&A, 397, 133

Russeil, D., Pestalozzi, M., Mottram, J. C., et al. 2011, A\&A, 526, A151

Schneider, N., Bontemps, S., Simon, R., et al. 2006, A\&A, 458, 855

Schuller, F., Menten, K. M., Contreras, Y., et al. 2009, A\&A, 504, 415

Silk, J. 1997, ApJ, 481, 703

Simon, R., Jackson, J. M., Clemens, D. P., Bania, T. M., \& Heyer, M. H. 2001, ApJ, 551, 747

Smith, L. F., Biermann, P., \& Mezger, P. G. 1978, A\&A, 66, 65

Solomon, P. M., Rivolo, A. R., Barrett, J., \& Yahil, A. 1987, ApJ, 319, 730

Spitzer, L. 1978, Physical processes in the interstellar medium (Wiley VCH)

Starck, J., \& Murtagh, F. 2006, Astronomical Image and Data Analysis (Springer)

Stil, J. M., Taylor, A. R., Dickey, J. M., et al. 2006, AJ, 132, 1158

Vázquez-Semadeni, E., Gómez, G. C., Jappsen, A. K., et al. 2007, ApJ, 657, 870

Watson, A. M., \& Hanson, M. M. 1997, ApJ, 490, L165

Wood, D. O. S., \& Churchwell, E. 1989, ApJ, 340, 265

Wu, H., Cao, C., Hao, C., et al. 2005, ApJ, 632, L79 


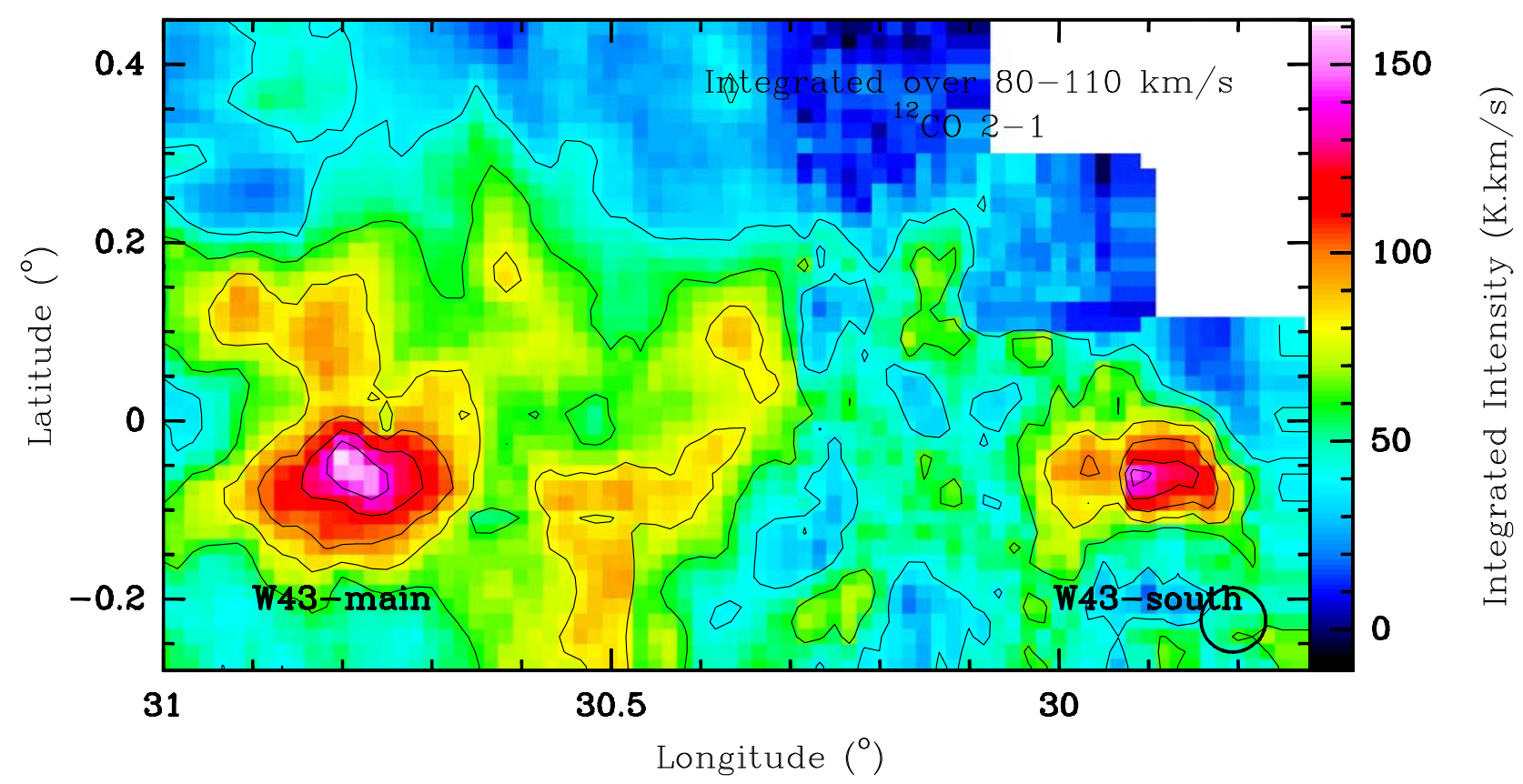

Fig. A.1. ${ }^{12} \mathrm{CO} 2-1$ intensity map of the inner part of the W43 molecular complex obtained with KOSMA. The lines are integrated over the $80-110 \mathrm{~km} \mathrm{~s}^{-1}$ velocity range. Contours go from to 40 to 140 by 20 in unit of $\mathrm{K} \mathrm{km} \mathrm{s}^{-1}$. The HPBW is plotted in the bottom-left corner.

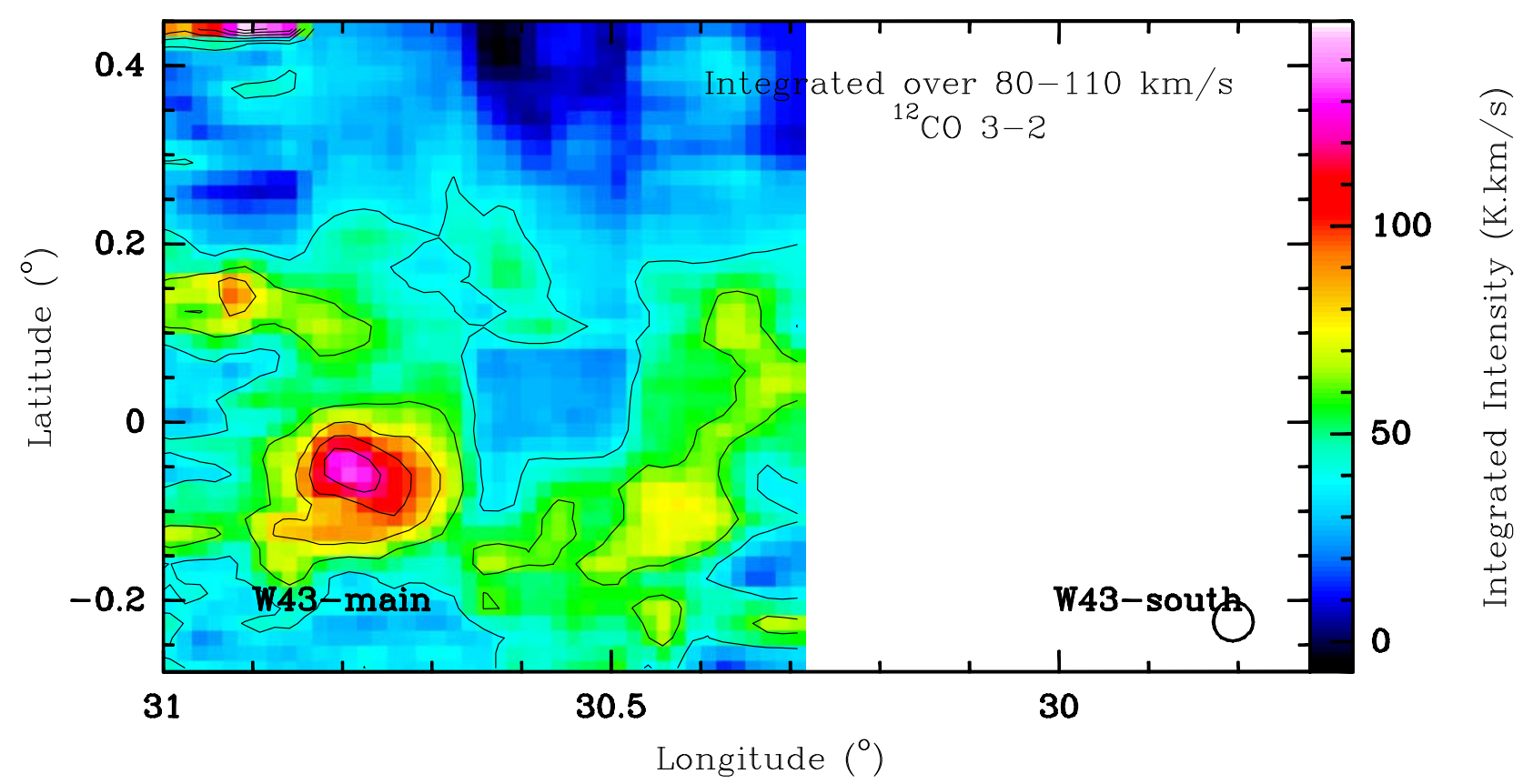

Fig. A.2. ${ }^{12} \mathrm{CO} 3-2$ intensity map of the inner part of the W43 molecular complex obtained with KOSMA. The lines are integrated over the $80-110 \mathrm{~km} \mathrm{~s}^{-1}$ velocity range. Contours go from to 40 to 140 by 20 in unit of $\mathrm{K} \mathrm{km} \mathrm{s}^{-1}$. The HPBW is plotted in the bottom-left corner. 


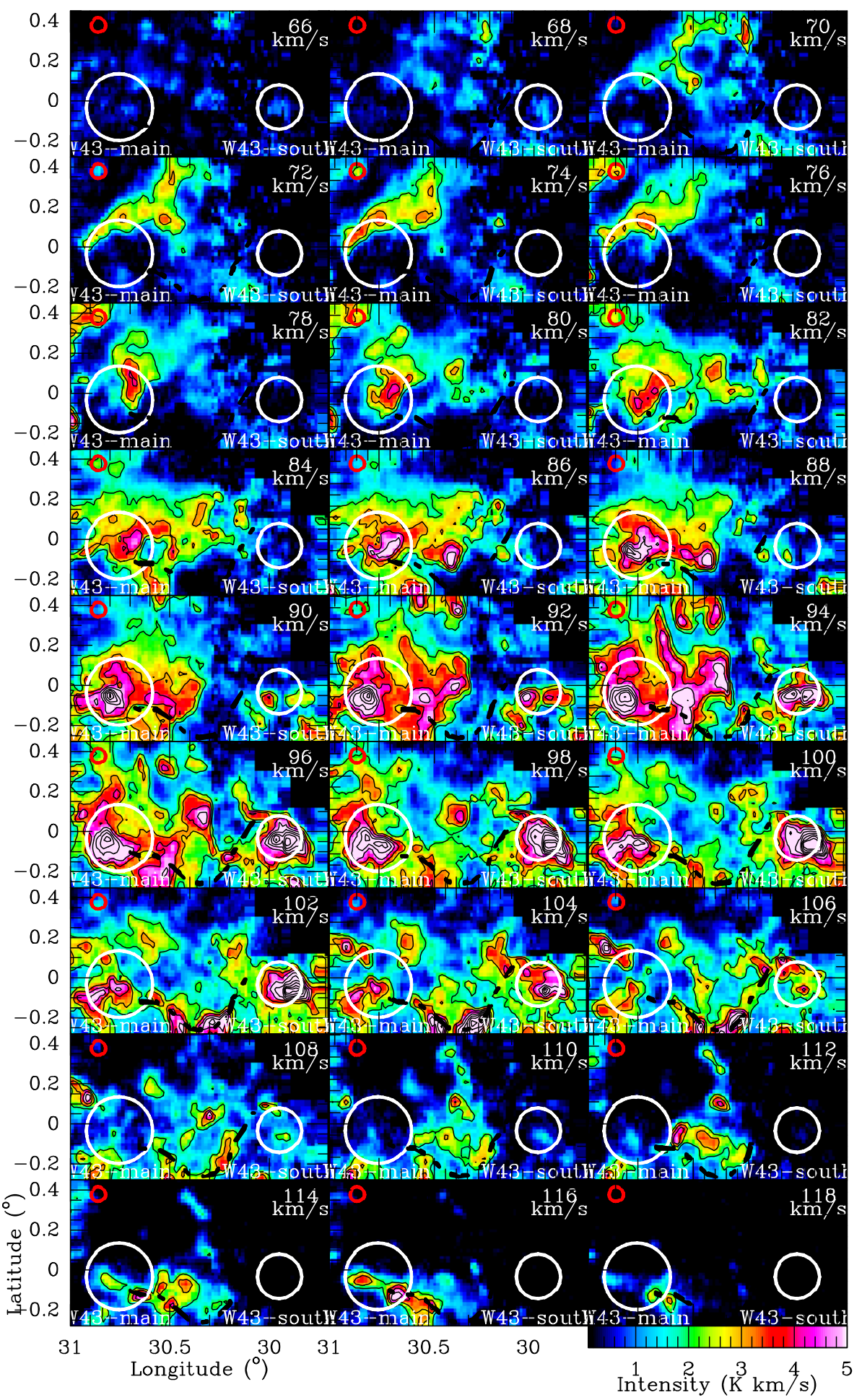

Fig. A.3. Velocity maps of the inner part of the W43 molecular complex in the ${ }^{12} \mathrm{CO} 2-1$ emission obtained with KOSMA, integrated over $\sim 2 \mathrm{~km} \mathrm{~s}^{-1}$-wide channels ranging from $V_{\mathrm{LSR}}=66$ to $118 \mathrm{~km} \mathrm{~s}^{-1}$. The W43-main and W43-south regions are indicated with white circles. The main molecular bridge linking them is outlined by a dashed curve. The HPBW is plotted in the top-left corner. 


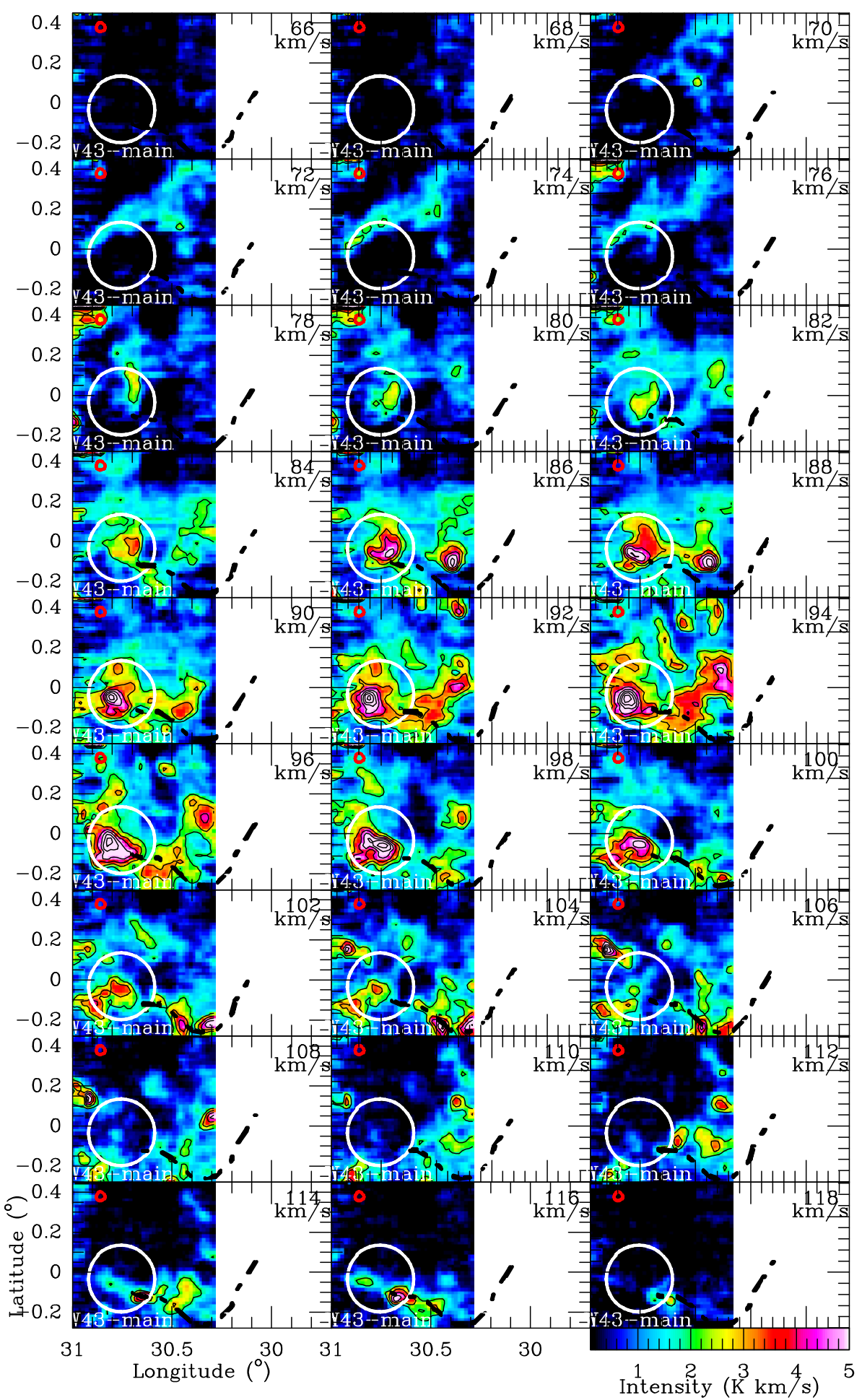

Fig. A.4. Velocity maps of the inner part of the W43 molecular complex in the ${ }^{12} \mathrm{CO} 3-2$ emission obtained with KOSMA, integrated over $\sim 2 \mathrm{~km} \mathrm{~s}^{-1}$-wide channels ranging from $V_{\mathrm{LSR}}=66$ to $118 \mathrm{~km} \mathrm{~s}^{-1}$. The W43-main and W43-south regions are indicated with white circles. The main molecular bridge linking them is outlined by a dashed curve. The HPBW is plotted in the top-left corner. 